\title{
PIAS1 and TIF1Y collaborate to promote SnoN SUMOylation and suppression of epithelial-mesenchymal transition
}

\author{
Ayan Chanda ${ }^{1} \cdot$ Yoshiho Ikeuchi $^{2,3} \cdot$ Kunal Karve $^{1} \cdot$ Anusi Sarkar ${ }^{1} \cdot$ Amrita Singh Chandhoke $^{1,4} \cdot$ Lili Deng $^{1} \cdot$ \\ Azad Bonni $\varpi^{2}$. Shirin Bonni ${ }^{1}$
}

Received: 18 February 2020 / Accepted: 20 July 2020 / Published online: 7 August 2020

(c) The Author(s), under exclusive licence to ADMC Associazione Differenziamento e Morte Cellulare 2020

\begin{abstract}
SUMO E3 ligases specify protein substrates for SUMOylation. The SUMO E3 ligases PIAS1 and TIF1 $\gamma$ target the transcriptional regulator SnoN for SUMOylation leading to suppression of epithelial-mesenchymal transition (EMT). Whether and how TIF1 $\gamma$ and PIAS1 might coordinate SnoN SUMOylation and regulation of EMT remained unknown. Here, we reveal that SnoN associates simultaneously with both TIF1 $\gamma$ and PIAS1, leading to a trimeric protein complex. Hence, PIAS1 and TIF1 $\gamma$ collaborate to promote the SUMOylation of SnoN. Importantly, loss of function studies of PIAS1 and TIF1 $\gamma$ suggest that these E3 ligases act in an interdependent manner to suppress EMT of breast cell-derived tissue organoids. Collectively, our findings unveil a novel mechanism by which SUMO E3 ligases coordinate substrate SUMOylation with biological implications.
\end{abstract}

\section{Introduction}

Conjugation of proteins with the small ubiquitin like modifier (SUMO) protein bears important consequences for protein substrates, regulating their biochemical, biological, and pathological properties [1,2]. SUMOylation is a multistep process involving the SUMO E1 activating enzyme, the SUMO E2 conjugating enzyme Ubc9, and a SUMO E3 ligase [3, 4]. SUMO E3 ligases play a key role in specifying

\section{Edited by M. Bianchi}

Supplementary information The online version of this article (https:// doi.org/10.1038/s41418-020-0599-8) contains supplementary material, which is available to authorized users.

$\triangle$ Shirin Bonni

sbonni@ucalgary.ca

1 Department of Biochemistry and Molecular Biology, Arnie Charbonneau Cancer Institute, Cumming School of Medicine, University of Calgary, Calgary, AB, Canada

2 Department of Neuroscience, Washington University School of Medicine, St. Louis, MO 63110, USA

3 Present address: Institute of Industrial Science, The University of Tokyo, Tokyo, Japan

4 Present address: Fog Pharmaceutical, Cambridge Discovery Park, Cambridge, MA 02140, USA protein substrates for SUMOylation [5], but the mechanisms underlying specifying SUMOylation remain incompletely understood.

The protein inhibitors for activated stats (signal transducer and activator of transcription) (PIASs) have been the subject of intense investigation [6]. Members of the PIAS SUMO E3 ligases, PIAS1-4, specify a diverse set of proteins for SUMOylation [6, 7]. Interestingly, some of the SUMO substrates regulated by the PIAS family of SUMO E3 ligases have been demonstrated to be also targeted by other SUMO E3 ligases [4, 8-10]. However, it is not clear how the function of distinct SUMO E3 ligases targeting common protein substrates for SUMOylation is coordinated.

The transcriptional regulator SnoN exerts diverse biological responses in various cell types including proliferating and postmitotic cells $[4,11-15]$. SnoN function is regulated by SUMOylation $[4,8,13]$. The SUMO E3 ligase PIAS1 associates with and promotes the SUMOylation of SnoN [4]. Interestingly, recent studies have revealed that TIF $1 \gamma$ interacts with and promotes SUMOylation of the SnoN1 isoform $[8,16]$. SUMOylation mediates the ability of SnoN to suppress epithelial-mesenchymal transition (EMT), a fundamental cellular process in development that is deregulated in disease states including cancer $[4,8,13,16,17]$. That PIAS1 and TIF1 $\gamma$ modify SnoN to suppress EMT raises the key question of whether PIAS 1 and TIF1 $\gamma$ act independently or collaboratively in promoting SUMOylation of SnoN and regulating EMT. 
In this study, we have uncovered that SnoN triggers the assembly of a PIAS1-SnoN-TIF1 $\gamma$ - multiprotein complex that promotes SnoN SUMOylation. Accordingly, PIAS1 and TIF1 $\gamma$ require each other to suppress EMT in threedimensional organoids of non-transformed and transformed mammary epithelial cells.

\section{Material and methods}

\section{Plasmids}

CMV-based plasmids expressing FLAG- or HA-tagged wildtype TIF1 $\gamma$, FLAG-TIF1 $\gamma$ CS, in which Cysteines 125 and 128 within the ring domain are converted to two serine residues, FLAG-TIF1 $\gamma \Delta \mathrm{C}$, in which the $\mathrm{C}$ terminal starting from amino acid 889 is deleted, FLAG-PIAS1, FLAG-PIAS1CS, in which Cysteine 350 is converted to a serine residue, FLAG-SnoN, HA-SnoN, MYC-SnoN, FLAG-SnoNres, green fluorescence protein (GFP) in fusion with the TIF1 $\gamma$ interaction peptide motif (TIPtide) in SnoN1 corresponding to amino acids 452-467, are described [8, 9, 13, 15, 17, 18]. Mouse U6 RNA polymerase III promoter-based RNA interference-CMV-GFP plasmids, co-expressing enhanced green fluorescent protein (EGFP), to express short hairpin (sh) RNAs targeting TIF1 $\gamma$, PIAS1, and SnoN are described [4, 8, 13, 18]. Restriction endonuclease-digested SnoN2, SnoNKdR, and PIAS1 were used as inserts to ligate with Restriction endonuclease-digested Renilla Luciferase (RLuc) tagged SnoN (RLuc-SnoN) vector [19], to generate plasmids encoding the proteins RLuc-SnoN2, RLuc-SnoNKdR, and RLuc-PIAS1, respectively. A pCAGip vector encoding a puromycin resistance marker alone or together with SnoNKdR, SUMO-SnoN, PIAS1, or PIAS1CS as a bicistronic transcript containing an internal ribosomal entry site was used to stably express the resistance marker alone (vector control) or together with the specific protein of interest, respectively, in cells $[4,18]$.

\section{Cell lines and transfections}

The human embryonic kidney 293T epithelial cell, human MDA-MB-231 breast cancer cells, and NAMRU murine mammary gland (NMuMG) epithelial cells were cultured in Dulbecco's modified Eagle's medium with high glucose and L-glutamine (Invitrogen, Canada) supplemented with $10 \%$ fetal bovine serum (FBS, Invitrogen), and $10 \mathrm{mg} / \mathrm{ml}$ recombinant human insulin (Invitrogen, Canada) in the case of the NMuMG cells. The human MCF7 breast cancer cells were cultured in minimum essential medium (Invitrogen, Canada) supplemented with $1 \times$ nonessential amino acids (Invitrogen, Canada), $1 \times$ sodium pyruvate (Invitrogen, Canada), $10 \% \mathrm{FBS}$, and $10 \mathrm{mg} / \mathrm{ml}$ recombinant human insulin. The 293T, MDA-MB231, NMuMG, and MCF7 cells were from the American Tissue Cell Collection. Cells were maintained in a humidified incubator at $37^{\circ} \mathrm{C}$ in $95 \%$ air and 5\% CO2 and all the cells were confirmed to be free of pathogenic mycoplasma strains by a PCR-ELISA kit. $293 \mathrm{~T}$ were transfected by the calcium-phosphate precipitation method [20]. The MDA-MB-231, NMuMG, and MCF7 cells were transfected using Lipofectamine 3000 reagent (Invitrogen, Canada) as per the manufacturer's instructions.

\section{Co-immunoprecipitation analyses}

Thirty percent confluent cells were transfected with expression plasmids as indicated. Two days post transfection, cells were lysed in TNTE (50 mM Tris, $150 \mathrm{mM} \mathrm{NaCl}$, and $1 \mathrm{mM}$ EDTA) buffer-containing $0.5 \%$ Triton $\mathrm{X}-100$, protease inhibitors, and phosphatase inhibitors [8, 17, 21]. Cell extracts were centrifuged at $14,000 \times g$ for $10 \mathrm{~min}$ at $4{ }^{\circ} \mathrm{C}$ and the supernatants were immunoprecipitated with mouse anti-FLAG (Sigma, Canada) or anti-HA (Biolegend, USA) antibodies. The input and immunoprecipitated protein complexes were resolved using SDS-PAGE followed by transfer to nitrocellulose membranes. The membranes were incubated with mouse anti-FLAG, mouse anti-HA, mouse anti-GFP (Santa Cruz, Canada), rabbit anti-PIAS1 (Abcam, USA), rabbit anti-SnoN (Proteintech, USA), mouse antiTIF1 $\gamma$ (Santa Cruz, Canada) or mouse anti-actin (Santa Cruz, Canada), as the primary antibody, and HRPconjugated goat anti-mouse or donkey anti-rabbit IgG (Jackson Laboratories) as secondary antibodies. Immunoprecipitates and total protein lysates containing RLuc alone or in fusion with SnoN or PIAS1 were subjected to RLuc assays using the RLuc kit (Promega, USA) and the Orion II luminometer (Berthold Detection Systems, Germany) detection system. Prior to RLuc analysis, immunoprecipitates were resuspended in TNE buffer-containing $0.1 \%$ Triton X-100 [19]. In the sequential immunoprecipitation assay, TIF1 $\gamma$ was immunoprecipitated using anti-FLAG antibody followed by elution of the FLAG immunocomplex using FLAG elution peptide (Millipore Sigma, Canada). The eluate was further subjected to anti-HA immunoprecipitation [22].

\section{In vivo SUMOylation assays}

Thirty percent confluent cells were transfected with a control vector, or a plasmid encoding MYC-SnoN, HA-hSUMO1 (referred to as SUMO hereafter), alone or together, without or with plasmids to express or knockdown TIF1 $\gamma$ or PIAS1 as shown. Two days post transfection, cells were lysed in TNTE (50 mM Tris, $150 \mathrm{mM} \mathrm{NaCl}$, and $1 \mathrm{mM}$ EDTA) buffer-containing $0.5 \%$ Triton $\mathrm{X}-100,0.1 \%$ SDS, protease 
inhibitors, and phosphatase inhibitors, alone or together with $20 \mathrm{mM}$ of n-ethylmaleimide (NEM) (Calbiochem, Canada), an isopeptidase inhibitor to suppress the activity of the SENP deSUMOylases upon lysis to help preserve the abundance of SUMOylated species of SnoN [4, 8, 17, 21]. The cell extracts were centrifuged at $14,000 \times g$ for $10 \mathrm{~min}$ at $4{ }^{\circ} \mathrm{C}$ and the supernatant was immunoprecipitated using mouse anti-MYC antibody (Santa Cruz, Canada) at $4{ }^{\circ} \mathrm{C}$, after saving $10 \%$ of the supernatant for Bradford protein quantification and protein expression analysis (Bio-Rad Laboratories, Canada). The immunoprecipitated sample and input lysates were immunoblotted with mouse anti-HA (Biolegend, Canada), rabbit anti-PIAS1 (Abcam, USA), rabbit anti-SnoN (Proteintech, USA), mouse anti-TIF1 $\gamma$ (Santa Cruz, Canada) or mouse anti-actin (Santa Cruz, Canada), as the primary antibody, and HRP-conjugated goat anti-mouse or donkey anti-rabbit IgG (Jackson Laboratories) as secondary antibodies, followed by Enhanced Chemiluminescence (ECL) (Millipore) reagent incubation and light signal detection using a VersaDoc 5000 Imager (Bio-Rad Laboratories). Densitometry was performed using Quantity One software (Bio-Rad Laboratories).

\section{Three-dimensional cultures}

Three-dimensional cultures of cells were prepared in 8-well chamber slides (Millicell EZ Slide, Millipore) or 96-well flat-bottom, ultra-low attachment plates (BD Biosciences, ON, Canada). 8-well chamber slide wells or each well of the 96-well dish were precoated with 75 or $50 \mu$, respectively, of $3 \mathrm{mg} / \mathrm{ml}$ final concentration Matrigel (BD Biosciences) cushions in antibiotic-antimycotic-containing growth medium. The Matrigel bed was allowed to solidify by incubating at $37{ }^{\circ} \mathrm{C}$ in $5 \% \mathrm{CO}_{2}$ containing tissue culture incubator. 700 or 400 cells per well of 8 -well chamber or 96-well dish respectively, were resuspended in 75 or $50 \mu$, respectively, of $5 \mathrm{mg} / \mathrm{ml}$ Matrigel and layered on top of the Matrigel bed and allowed to solidify by incubating at $37^{\circ} \mathrm{C}$ in $5 \% \mathrm{CO}_{2}$ containing tissue culture incubator. Complete medium was layered on top after $1 \mathrm{~h}$. The next day and every third day the three-dimensional cell-derived organoids received $100 \mathrm{pM}$ TGF, $10 \mu \mathrm{M} \mathrm{KI}$, alone or together until 8 days in culture. Differential interference contrast images of six representative organoids from each well were captured at $30 \times$ objective (Olympus IX70) following an overall estimate of the total number ( 50) and assessment of growth phenotypes (acini vs. filled for NMuMG and MCF7 organoids and nondeformed vs. deformed for MDA-MB-231 organoids).

\section{Indirect immunofluorescence}

The 3D-organoids were fixed with $4 \%$ formaldehyde, permeabilized using $0.5 \%$ ice-cold Triton X-100 solution for time, and blocked using 10\% BSA in phosphate-buffered saline at $37^{\circ} \mathrm{C}$ for $1 \mathrm{~h}$. The organoids were then subjected to indirect immunofluorescence staining using a rabbit Ecadherin antibody (Cell Signaling Technology, Canada) as the primary antibody and goat anti-rabbit antibody conjugated to Cy5 dye (Jackson Lab, Burlington, ON, Canada) as the secondary antibody along with the DNA fluorescent dye Hoechst 33342 (Invitrogen) to visualize cells' nuclei. Fluorescence images of the multicellular colonies were captured using a fluorescent microscope with a $40 \times$ objective lens (Olympus Fluoview FV1000 microscope, Canada). For each biological replicate, the exposure time for each Ecadherin and Hoechst-specific fluorescence signals was kept constant for all the different conditions tested. After an overall observation of each well, images of 2-3 colonies per experimental condition were captured.

\section{Statistical analyses}

Replicates are used to account for inter-experimental variations in measurements of a particular parameter, which may otherwise lead to erroneous hypothesis testing and parameter estimation. Biological replicates refer to the use of biologically distinct samples or cell culture populations in a specific type of experiment, and is used to alleviate random biological variations which may be a source of noise or be part of the subject of study [23]. A minimum of three biological replicates were used in each type of experiment in this study to facilitate statistical inference. The Student's $t$ test (for two groups) or oneway analysis of variance followed by Tukey-Kramer post test (for more than two groups) using InStat (Graphpad InStat, USA) were performed to evaluate the statistical significance of data from biological replicates per experiment where appropriate. Values of $P \leq 0.05$ were considered statistically significant. Data are presented graphically as mean \pm standard error of the mean (S.E.M.) for experiments with a minimum of three biological replicates.

\section{Results}

\section{SnoN promotes a TIF1Y-SnoN-PIAS1 multiprotein complex}

PIAS1 and TIF1 $\gamma$ promote SUMOylation of SnoN $[4,8,16]$. A key question raised by these studies is whether and how the activity of PIAS1 and TIF1 $\gamma$ as SUMO E3 ligases is coordinated. To address this question, we first determined if PIAS 1 and TIF1 $\gamma$ interact. Co-immunoprecipitation analyses showed that TIF1 $\gamma$ and PIAS1 formed a complex (Fig. 1a-d). SnoN associates with each of TIF1 $\gamma$ and 
A

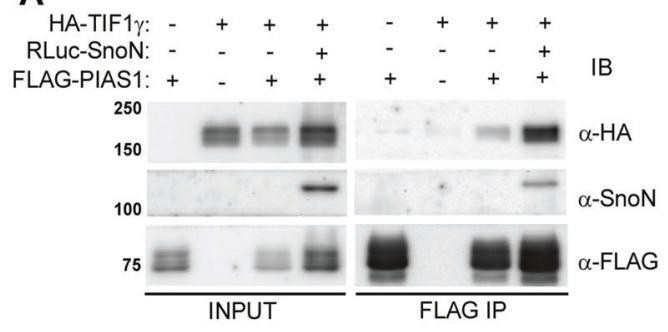

B

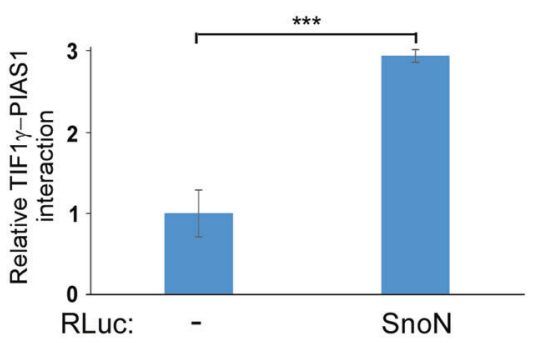

C

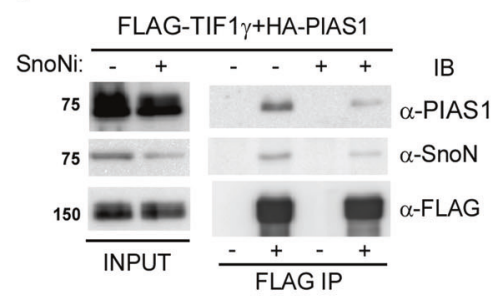

$\mathbf{F}$

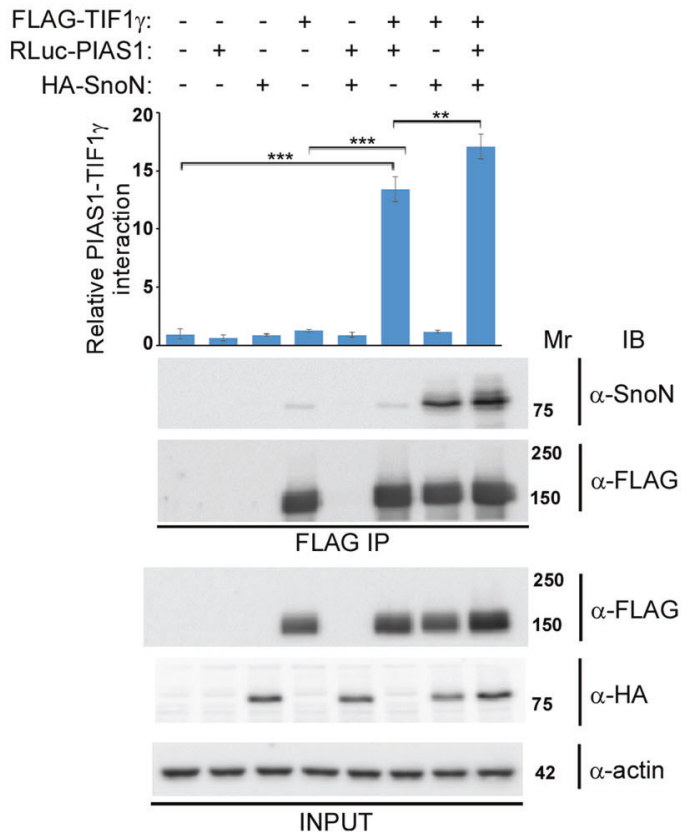

D

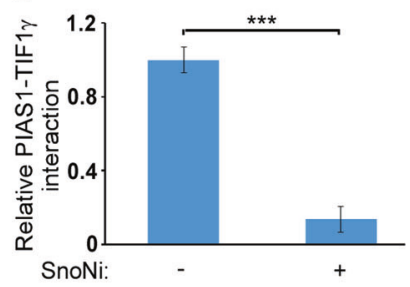

G

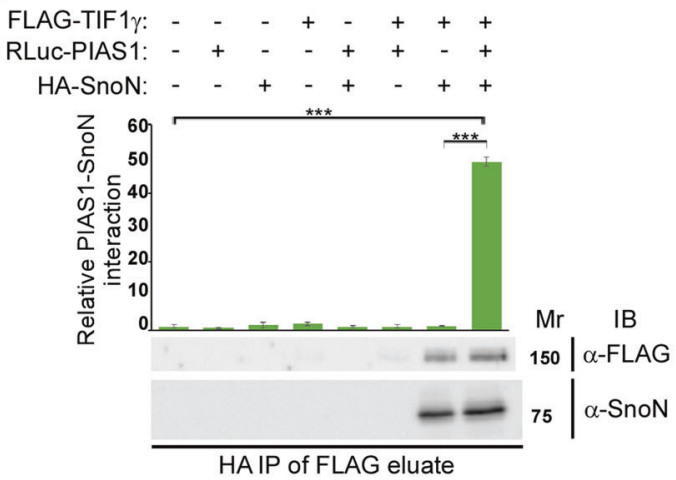

The finding that SnoN promoted the interaction between TIF1 $\gamma$ and PIAS1 raised the possibility of a TIF1 $\gamma$-SnoN-PIAS1 multiprotein complex. To address this question, we performed sequential co-immunoprecipitation assays. Lysates of cells transfected with the vector control or a plasmid expressing TIF1 $\gamma$, PIAS1, and SnoN in different combinations were subjected to TIF1 $\gamma$ immunoprecipitation ( $\alpha$-FLAG IP), followed by SnoN immunoprecipitation ( $\alpha$-HA IP) of the eluates of the TIF1 $\gamma$ immunocomplexes. PIAS1, expressed as a RLuc-tagged protein, in each of these two immunoprecipitations was quantified using Renilla luciferase activity [19, 24]. As expected, SnoN coimmunoprecipitated with TIF1 $\gamma$. The 
Fig. 1 SnoN promotes a TIF1 $\gamma$-SnoN-PIAS1-containing multiprotein complex. a Lysates of $293 \mathrm{~T}$ cells transfected with vector control or a plasmid containing cDNA encoding HA-TIF1 $\gamma$, FLAGPIAS1, alone or in combination, together with a plasmid containing cDNA encoding Renilla luciferase (RLuc) (-) alone, or RLuc-SnoN fusion $(+)$, were subjected to PIAS1 immunoprecipitation ( $\alpha$-FLAG IP) followed by TIF1 $\gamma(\alpha-\mathrm{HA})$, SnoN $(\alpha-\mathrm{SnoN})$, and PIAS1 ( $\alpha$-FLAG) immunoblotting (IB). Lysates were subjected to TIF1 $\gamma(\alpha-\mathrm{HA})$, SnoN $(\alpha-$-SnoN), and PIAS1 ( $\alpha$-FLAG) immunoblotting (IB) as the input. b Bar graph represents relative mean \pm SEM of coimmunoprecipitated TIF1 $\gamma$ normalized to the protein abundance of PIAS1 in the corresponding immunoprecipitated sample and TIF1 $\gamma$ in the input in the absence or presence of expressed SnoN from three biological replicates including from the data shown in the last two lanes of $1 \mathrm{~A}$ (Student's $t$ test: $* * * P \leq 0.001$ ). c Lysates of 293T cells transfected with HA-PIAS1 and FLAG-TIF1 $\gamma$ together with a control RNAi vector (-) or one expressing short hairpin RNA (shRNA) targeting SnoN (SnoNi), were subjected to TIF1 $\gamma$ immunoprecipitation using a mouse $\alpha$-FLAG antibody $(+)$ or a mouse $\operatorname{IgG}(-)$ as a negative control, followed by PIAS1 ( $\alpha$-PIAS1), SnoN $(\alpha-$ SnoN), and TIF1 $\gamma(\alpha-$ FLAG) immunoblotting (IB) (right hand side panel). Lysates were subjected to immunoblotting for TIF1 $\gamma(\alpha-\mathrm{FLAG})$, SnoN $(\alpha-$ SnoN), and PIAS1 ( $\alpha$-PIAS1) immunoblotting as the input (left hand side panel). d Bar graph represents relative mean \pm SEM of coimmunoprecipitated PIAS1, normalized to the protein abundance of immunoprecipitated TIF1 $\gamma$ and the input PIAS1, in the absence $(-)$ or presence $(+)$ of shRNA targeting endogenous SnoN (SnoNi), from four biological replicates including the one with the data shown in lanes 2 and 4 of right-hand side panel in $\mathbf{c}$ (Student's $t$ test: ***P 0.001). e Bar graph depicts relative input-normalized SnoN (mean \pm SEM, $n=3$ biological replicates) interacting with PIAS1 obtained by luciferase measurements of $80 \%$ of $\alpha$-FLAG immunoprecipitation (IP) of lysates of 293T cells co-expressing FLAG-PIAS1 and RLuc alone $(-)$, or in fusion with SnoN or SnoN2. Four percent of lysates (input) and $20 \%$ of the PIAS1 immunocomplexes were subjected to luciferase measurements and PIAS1 ( $\alpha$-FLAG) immunoblotting (IB), respectively. f Top panel: bar graph depicts relative input-normalized PIAS1 interaction with TIF $1 \gamma$ (mean \pm SEM, $n=4$ biological replicates) obtained by subjecting lysates of $293 \mathrm{~T}$ cells, transfected with control vector $(-)$ or one expressing FLAG-TIF1 $\gamma$, HA-SnoN, alone or combined, together with a plasmid expressing RLuc only $(-)$ or in fusion with PIAS1 $(+)$, to TIF1 $\gamma$ immunoprecipitation $(\alpha$-FLAG IP) followed by subjecting $10 \%$ of the immunocomplexes and $0.5 \%$ of the lysates to luciferase measurements, with the latter serving as the input values (ANOVA: $* * P \leq 0.01 ; * * * P \leq 0.001$ ). Lower panels: representative scans from one of the biological replicates in which $10 \%$ of the TIF1 $\gamma$ immunocomplexes were subjected to TIF $1 \gamma(\alpha$-FLAG) and SnoN $(\alpha-$-SnoN) immunoblotting (IB), and where lysates were subjected to TIF1 $\gamma(\alpha-$ FLAG), SnoN $(\alpha-$ SnoN), and actin ( $\alpha$-actin) immunoblotting as the input, with the latter as a loading control. g Upper panel: bar graph depicts relative input-normalized PIAS1 interaction with SnoN (mean $\pm \mathrm{SEM}, n=4$ biological replicates) obtained by SnoN immunoprecipitation $(\alpha-\mathrm{HA})$ of FLAG peptide eluates of the TIF1 $\gamma$ (FLAG) immunocomplexes of cells transfected and immunoprecipitated as described in a, followed by subjecting the SnoN immunocomplexes to luciferase measurements, with the input from a serving as the input values (ANOVA: $* * * P \leq 0.001$ ). Lower panels: representative scans from one of the three biological replicates showing TIF1 $\gamma(\alpha-$ FLAG) and SnoN $(\alpha-$ SnoN) immunoblotting (IB) of $10 \%$ of the SnoN immunocomplexes.

proportion of PIAS1 in TIF1 $\gamma$ immunocomplexes was enhanced by increased abundance of SnoN in these immunocomplexes (Fig. 1f). Importantly, PIAS1 and TIF1 $\gamma$

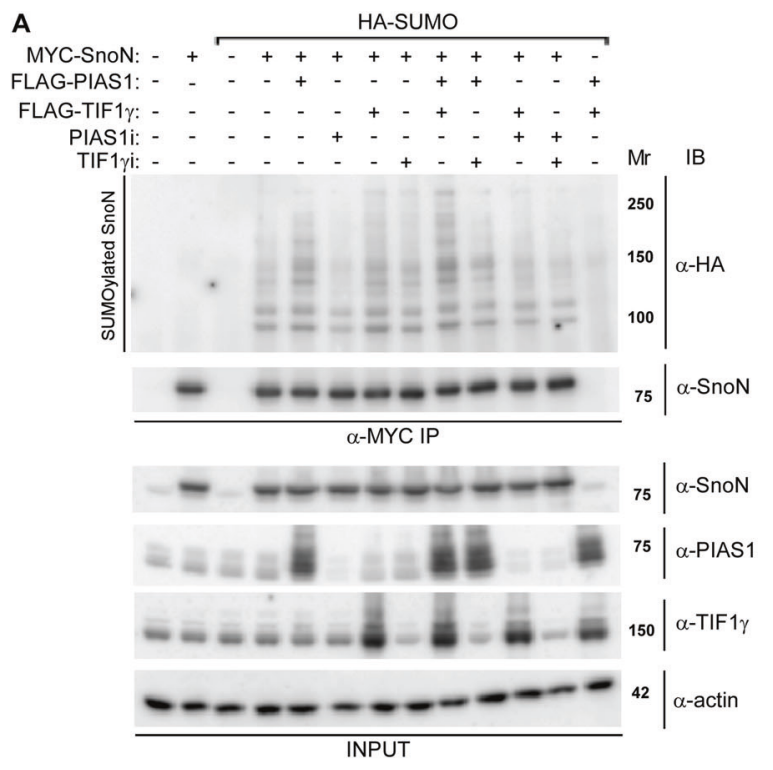

B

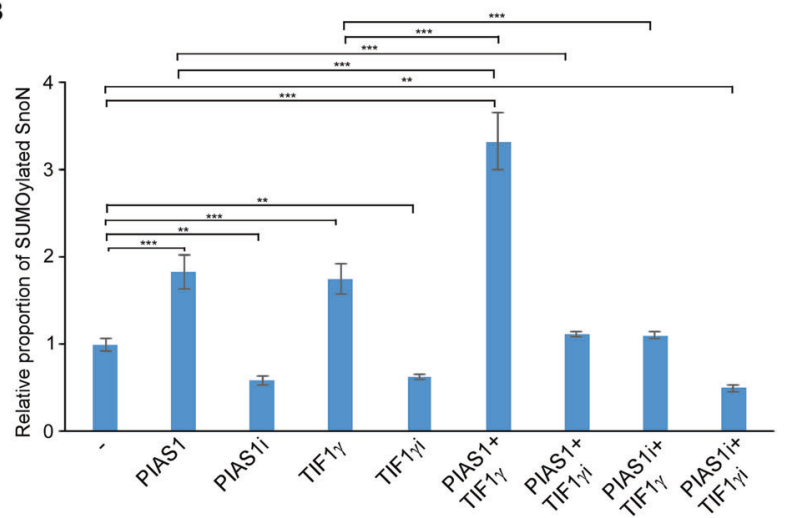

Fig. 2 A PIAS1-TIF1 $\gamma$ protein complex that promotes SnoN SUMOylation. a NEM-treated lysates of 293T cells transfected with control plasmids, alone or together with plasmids encoding MYCSnoN or HA-SUMO, alone or together, alone or along with different combinations of plasmids encoding FLAG-TIF1 $\gamma$, FLAG-PIAS 1 , short hairpin RNA (sh) targeting PIAS1 (PIAS1i), or shRNA targeting TIF1 $\gamma$ (TIF1 $\gamma \mathrm{i})$, were subjected to SnoN immunoprecipitation $(\alpha-$ MYC IP) followed by SUMO ( $\alpha-\mathrm{HA})$ and SnoN ( $\alpha-\mathrm{SnoN})$ immunoblotting. Lysates were subjected to TIF1 $\gamma$, SnoN, PIAS1, and actin immunoblotting as the input, with the latter as a loading control. b The bar graph represents the mean \pm SEM of proportion of SUMOylated SnoN relative to unmodified SnoN quantified from HA and SnoN immunoblots of SnoN immunoprecipitation and expressed relative to the proportion of SUMOylated SnoN in lysates of cells transfected with $\mathrm{MYC} / \mathrm{SnoN}$ and HA/SUMO along with empty vectors as a control (lane 4). The data are from three biological replicates including from the replicate whose data are shown in a (ANOVA: $* * P \leq 0.01$; $* * * P \leq 0.001)$.

coimmunoprecipitated with SnoN in TIF1 $\gamma$ immunocomplex-containing eluates supporting the existence of a PIAS1-SnoN-TIF1 $\gamma$ trimeric complex (Fig. 1g). Collectively, these data suggest that SnoN supports the assembly of PIAS1-SnoN-TIF1 $\gamma$ multimeric protein complex in mammalian cells. 
A

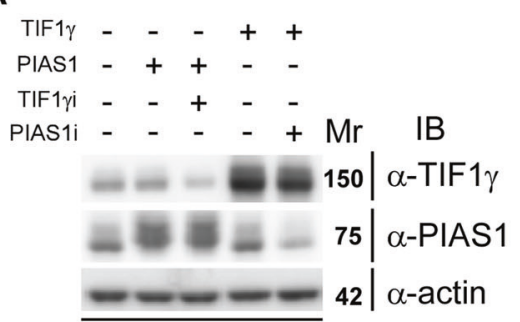

Cell lysates

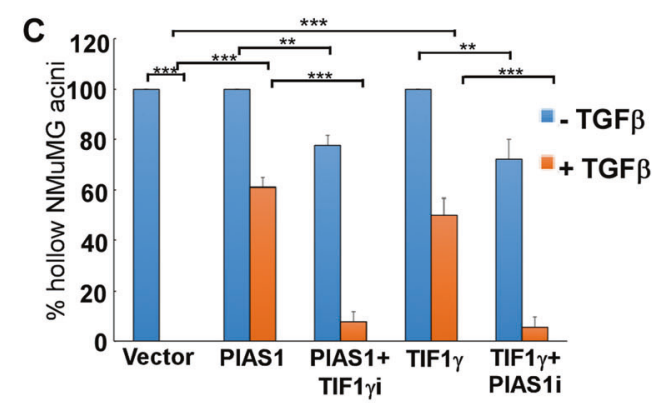

E
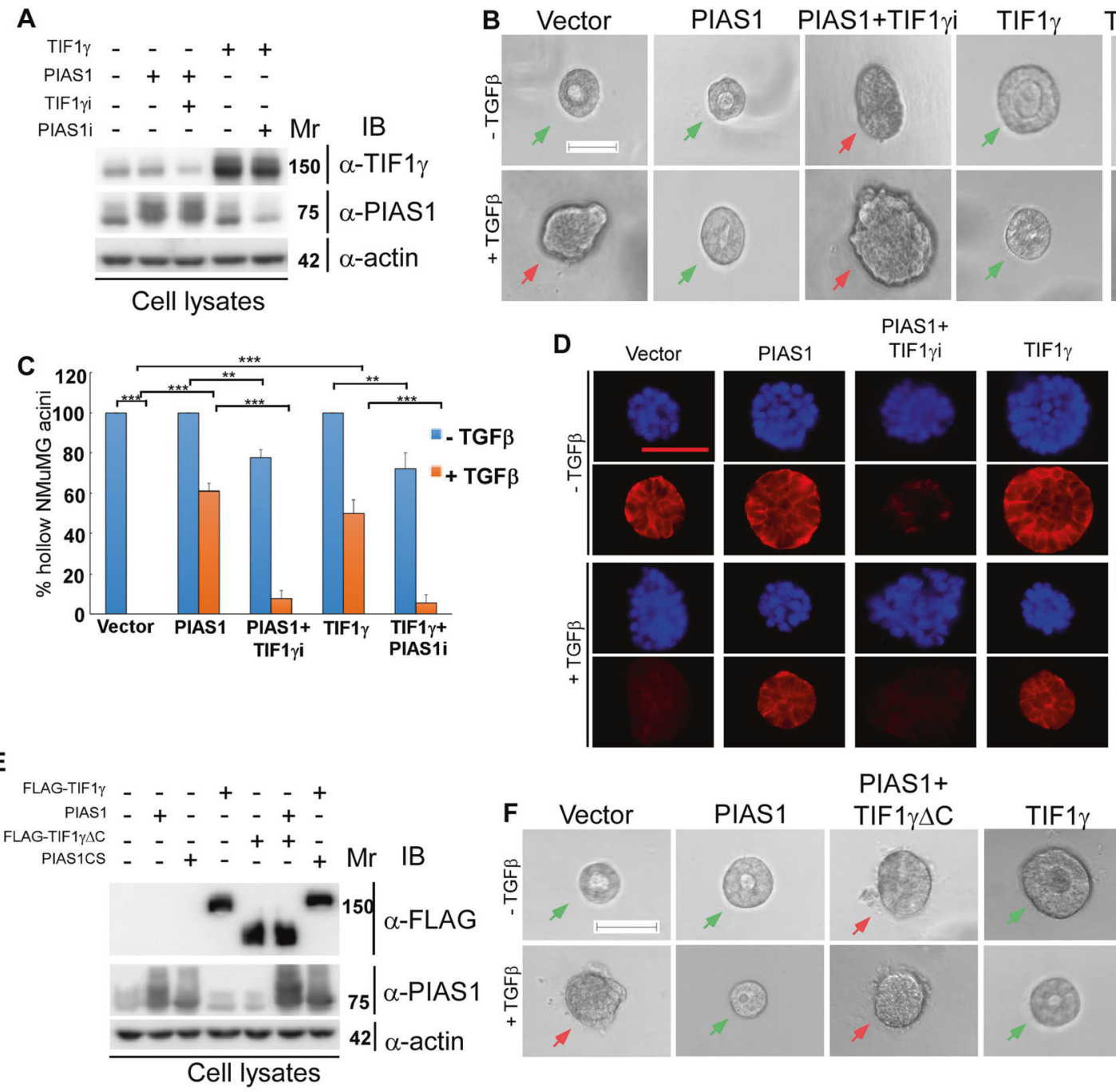

D Vector
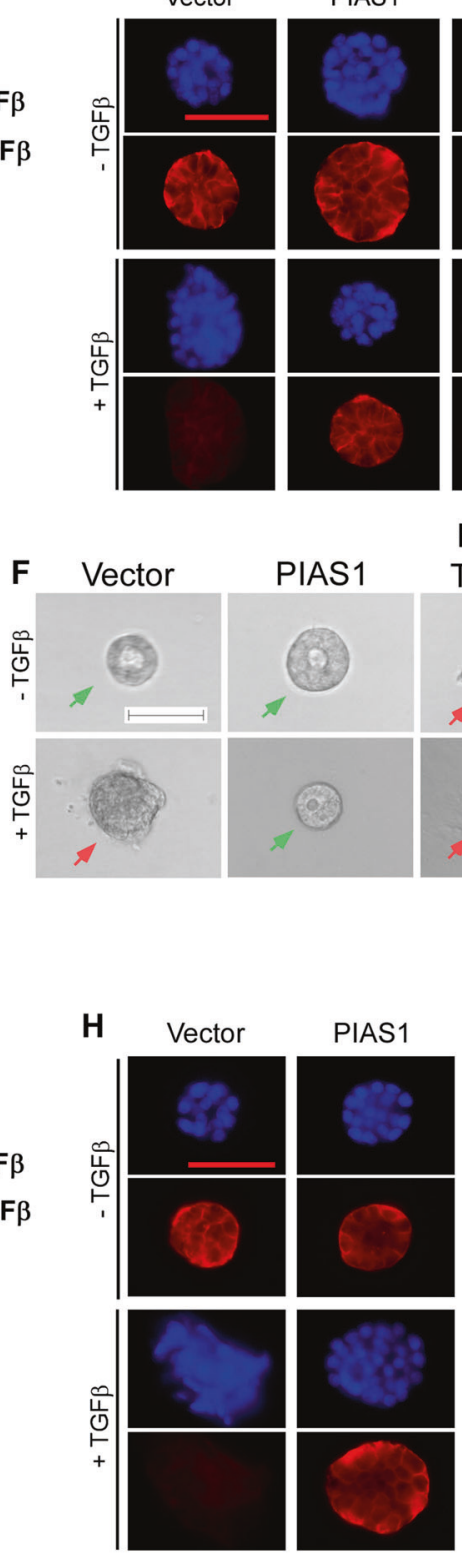

PIAS1+
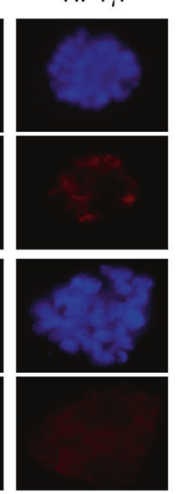

PIAS1+ $\mathrm{TIF} 1 \gamma \Delta \mathrm{C}$
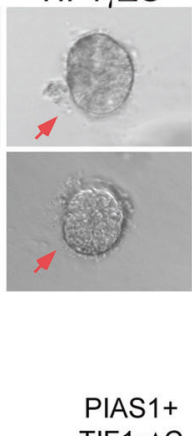
$\mathrm{TIF} 1 \gamma \Delta \mathrm{C}$
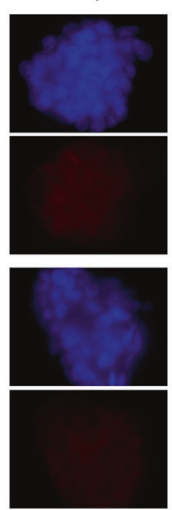

TIF $1 \gamma+P I A S 1 i$

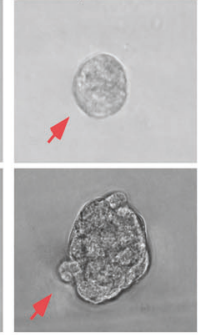

TIF $1 \gamma^{+}$
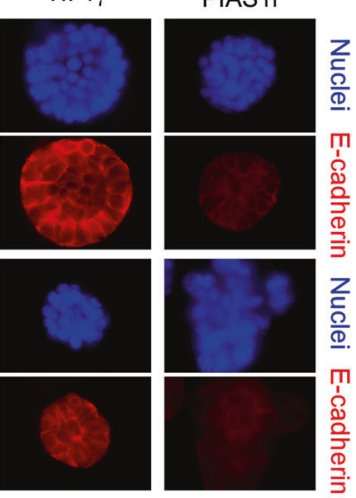

$\mathrm{TIF} 1 \gamma+$ PIAS1CS
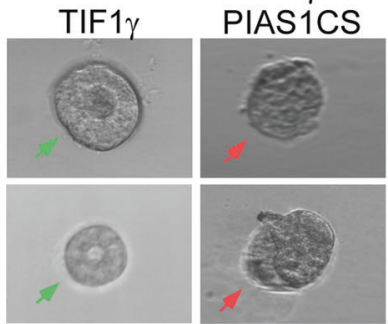

G

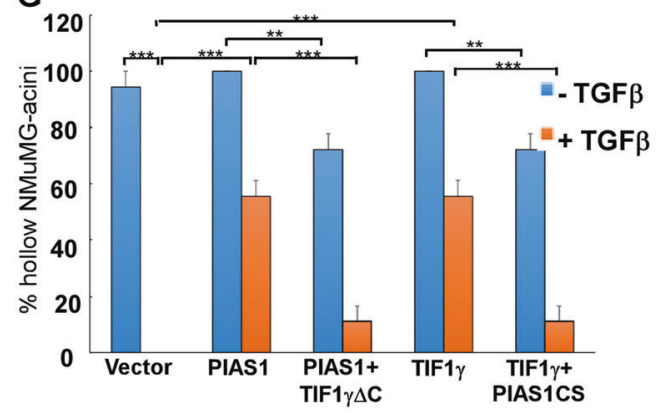

\section{PIAS1 and TIF1Y cooperate to regulate SnoN SUMOylation}

We next performed in vivo SUMOylation assays to address the question of the functional significance of formation of the
PIAS1-SnoN-TIF1 $\gamma$ protein complex $[4,17]$. As expected, coexpression of SnoN with SUMO led to NEM-sensitive SUMO-immunoreactive protein species in SnoN immunoprecipitations (Fig. 2a, b) [4]. Expression of exogenous PIAS1 or TIF1 $\gamma$ increased the proportion of SUMOylated 
Fig. 3 PIAS1 and TIF1 $\gamma$ act reciprocally to suppress EMT in mammary epithelial cell-derived organoids. a PIAS1, TIF $1 \gamma$, and actin immunoblotting of lysates of NMuMG cells expressing PIAS1, TIF1 $\gamma$, PIAS1i, and TIF1 $\gamma$ i alone or together, and which were used to generate three-dimensional organoids shown in b-d. b Representative DIC light microscopy micrographs of untreated or 100pM TGF $\beta$-treated 8-day-old organoids derived from NMuMG cells transfected and assessed as in a. Green and red arrows indicate acinar and filled organoids, respectively. c Bar graph depicts mean \pm SEM proportion of acinar organoids expressed as a percentage of total colonies counted for each experimental condition from three biological replicates including the replicates with data shown in b. d Representative fluorescence microscopy scans of E-cadherin-(red) and nuclei-(blue) stained fixed 8day-old three-dimensional organoids derived from NMuMG cells transfected and assessed in a, b. e PIAS1, FLAG, and actin immunoblotting of lysates of NMuMG cells expressing PIAS1, FLAG-TIF1 $\gamma$, PIAS1CS, and FLAG-TIF $1 \gamma \Delta \mathrm{C}$ alone or together, and which were used to generate three-dimensional organoids shown in $\mathbf{f}-\mathbf{h}$. $\mathbf{f}$ Representative DIC light microscopy micrographs of untreated or 100pM TGF $\beta$-treated 8-day-old organoids derived from NMuMG cells transfected and assessed as in e. Green and red arrows indicate acinar and filled organoids, respectively. $\mathrm{g}$ Bar graph depicts mean \pm SEM proportion of acinar organoids expressed as a percentage of total colonies counted for each experimental condition from three biological replicates including the replicates with data shown in $\mathbf{f}$. h Representative fluorescence microscopy scans of E-cadherin-(red) and nuclei-(blue) stained fixed 8day-old three-dimensional organoids derived from NMuMG cells transfected and assessed in e, f. Statistical difference, ANOVA: $* * P \leq$ $0.01 ; * * * P \leq 0.001$. Scale bar indicates $50 \mu \mathrm{m}$.

SnoN (Fig. 2a, b). Combined expression of PIAS1 and TIF1 $\gamma$ further enhanced the proportion of SUMOylated SnoN (Fig. 2a, b). Knockdown of endogenous PIAS1 or TIF1 $\gamma$ reduced the proportion of SUMOylated SnoN in cells (Fig. 2a, b). Knockdown of endogenous TIF1 $\gamma$ and PIAS1 led to reciprocal reduction of the ability of expressed PIAS1 and TIF1 $\gamma$ to promote the proportion of SUMOylated SnoN (Fig. 2a, b), suggesting that PIAS1 and TIF1 $\gamma$ cooperate to promote SUMOylation of SnoN. Collectively, our data suggest that SnoN may act as an adapter to promote a TIF1 $\gamma$-PIAS1 complex to promote its own SUMOylation.

\section{PIAS1 and TIF1Y regulate morphogenesis of mammary epithelial organoids}

The finding that SnoN promotes a TIF1 $\gamma$-PIAS1 SUMO E3 ligase complex raised the question of the biological significance of the interaction. EMT is a fundamental cellular process in tissue and organ development and contributes to homeostasis $[25,26]$. The secreted protein transforming growth factor beta (TGF $\beta$ ) induces EMT in the developing and adult organism $[27,28]$. Both PIAS1 and TIF1 $\gamma$ act via SUMOylated SnoN to suppress TGF $\beta$-induced EMT, raising the question of whether PIAS1-TIF1 $\gamma$ reciprocally regulate each other's role in EMT. NMuMG murine mammary epithelial cells represent a widely used model to investigate EMT. Three-dimensional culture approaches, where cells are grown in the context of an extracellular matrix (ECM) support system, provide suitable in vivo insight on the regulation of cellular processes like EMT [29]. Isolated non-transformed epithelial cells, e.g., NMuMG cells, cultured in the context the ECM components contained in the Matrigel, replicate forming aggregates that organize into multicellular spherical organoids with hollow centers or lumens (Fig. S1B) [8, 21]. A key feature of these acini is the apical (toward lumen) to basal (towards the ECM) polarity, and which is lost in EMT. EMT-related changes manifest as lumen filling and may include budding and deformation of epithelial cell-derived organoids $[8,21]$. The epithelial marker E-cadherin in these epithelial cell-derived organoids localizes at the basolateral junction (Fig. S1D). A hallmark of EMT is the mislocalization and loss of E-cadherin, which can be detected by indirect immunofluorescence $[4,8,21]$.

We used a Matrigel-based three-dimensional culture system, to address the question of whether TIF1 $\gamma$ and PIAS1 coordinately regulate TGF $\beta$-induced EMT $[8,21]$. PIAS1 suppression of TGF $\beta$-induced EMT has only been reported in conventional two-dimensional cultures [4]. We, thus, first determined if PIAS1-SnoN SUMOylation axis regulates EMT in epithelial cell-derived organoids. We found that expression of exogenous PIAS1 suppressed TGF $\beta$-induced acini filling and deformation of threedimensional NMuMG cell-derived organoids, which was reversed by expression of the SUMO-loss of function SnoNKdR (Fig. S1A-C). Conversely, expression of the SUMO E3 ligase inactive PIASCS promoted acini filling and deformation of the organoids even in the absence of TGF $\beta$, and which was reversed by expression of the SUMO gain of function SUMO-SnoN, a stable linear protein fusion of SUMO and SnoN (Fig. S1E-G). Indirect immunofluorescence analyses demonstrated that PIAS1 acts via SUMOylated SnoN to suppress TGF $\beta$-induced mislocalization and loss of E-cadherin in NMuMG cellderived organoids (Fig. S1D, H). Together, these data show that similar to TIF1 $\gamma$ [8], PIAS1 suppresses TGF $\beta$-induced EMT in the mammary epithelial cell-derived organoids in a SUMOylated SnoN-dependent manner.

Next, we addressed the question of whether PIAS1 and TIF1 $\gamma$ coordinately suppress EMT in NMuMG cell-derived organoids. We found that knockdown of endogenous TIF1 $\gamma$ and PIAS1 abrogated the ability of exogenous PIAS1 and TIF1 $\gamma$, respectively, to suppress TGF $\beta$-induced EMT as assessed by acini filling, and E-cadherin loss and mislocalization of the organoids (Fig. 3a-d), suggesting that endogenous and exogenous PIAS1 and TIF1 $\gamma$ intersect to suppress TGF $\beta$-induced EMT in the mammary epithelialderived organoids. In other experiments, we found that expression of the SUMO E3 ligase inactive TIF1 $\gamma \Delta \mathrm{C}$, which is deleted in C-terminal PHD and Bromo domains, inhibited exogenous PIAS1 suppression of TGF $\beta$-induced 
EMT in NMuMG cell-derived organoids. Conversely, the SUMO E3 ligase inactive PIAS1CS reduced exogenous TIF1 $\gamma$ suppression of TGF $\beta$-induced EMT in the NMuMGderived organoids (Fig. 3e-h). Collectively, these data suggest that a TIF1 $\gamma$-PIAS1 complex acts in a SUMO E3 ligase-dependent manner to suppress EMT in epithelialderived organoids.
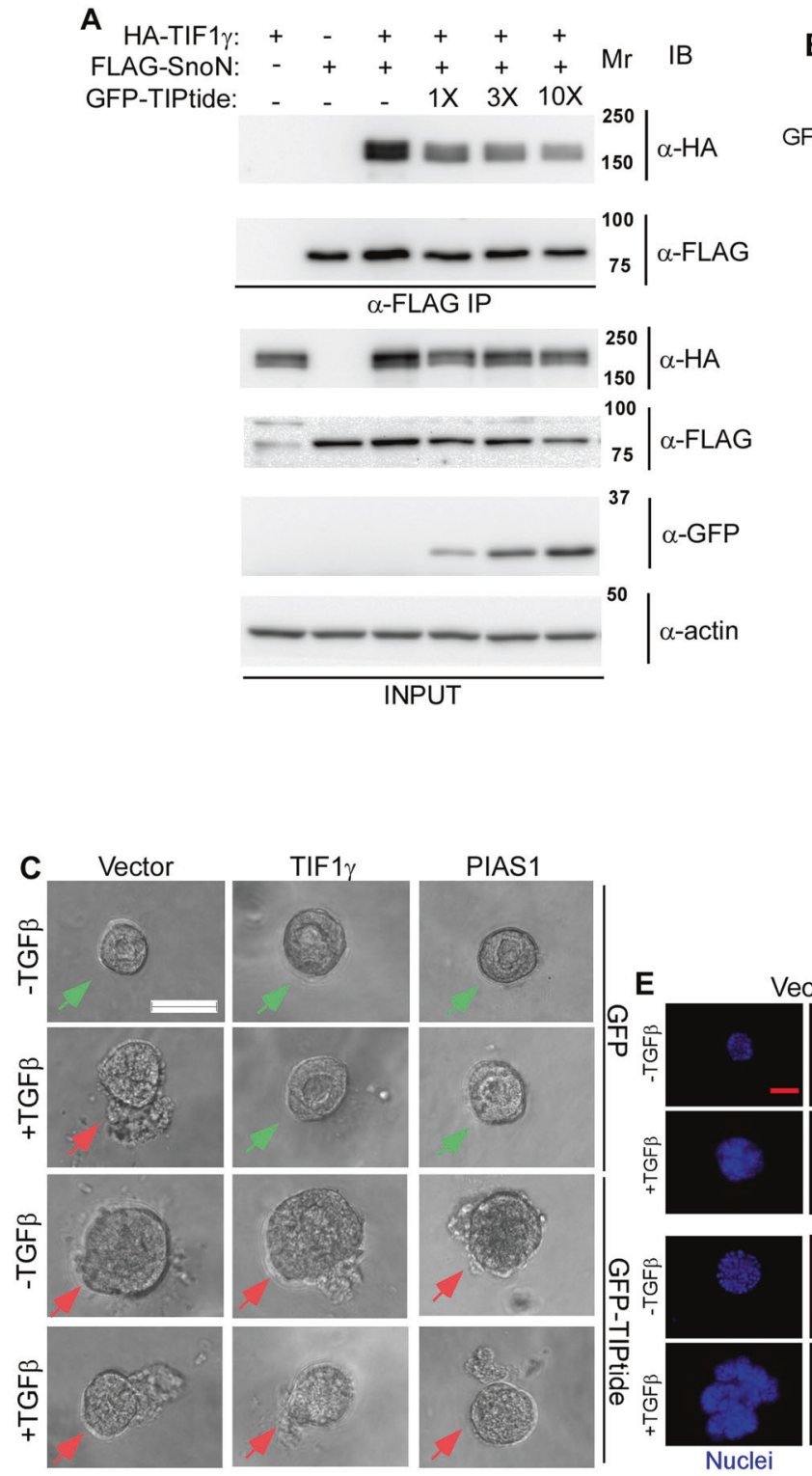

Fig. 4 PIAS1-TIF1 $\gamma$ SUMO E3 ligase act via SnoN to suppress TGF $\beta$-induced EMT in NMuMG cell-derived organoids. a Lysates of 293 T cells expressing HA-TIF1 $\gamma$, FLAG-SnoN, and GFP-TIPtide alone or together were subjected to SnoN immunoprecipitation (FLAG IP) followed by TIF1 $\gamma(\alpha-\mathrm{HA})$ and SnoN ( $\alpha$-FLAG) immunoblotting. Lysates were subjected to immunoblotting for TIF1 $\gamma(\alpha-\mathrm{HA})$, SnoN $(\alpha$-FLAG), TIPtide ( $\alpha$-GFP), and actin ( $\alpha$-actin) as the input, with the latter as a loading control. b TIF1 $\gamma$, PIAS1, GFP, and actin immunoblotting of lysates of NMuMG cells expressing TIF1 $\gamma$, PIAS1, and GFP-TIPtide alone or together, and which were used to generate threedimensional organoids shown in c-e. c Representative DIC light
Next, we addressed the question of whether SUMOylated SnoN plays a role in the TIF1 $\gamma$-PIAS1-mediated suppression of EMT. The TIPtide motif specifies SnoN interaction with TIF1 $\gamma$ but not with PIAS1 (Fig. 1e and [8]). We confirmed that TIPtide competes with SnoN for association with TIF1 $\gamma$ (Fig. 4a). Accordingly, we found that expression of TIPtide promoted EMT in NMuMG cell-

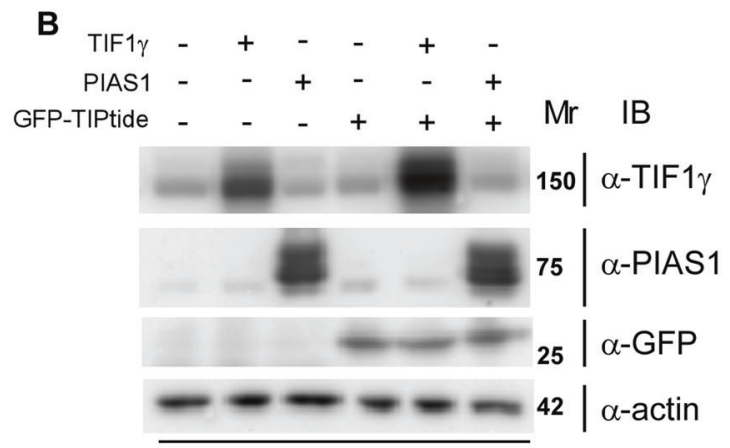

Cell lysates
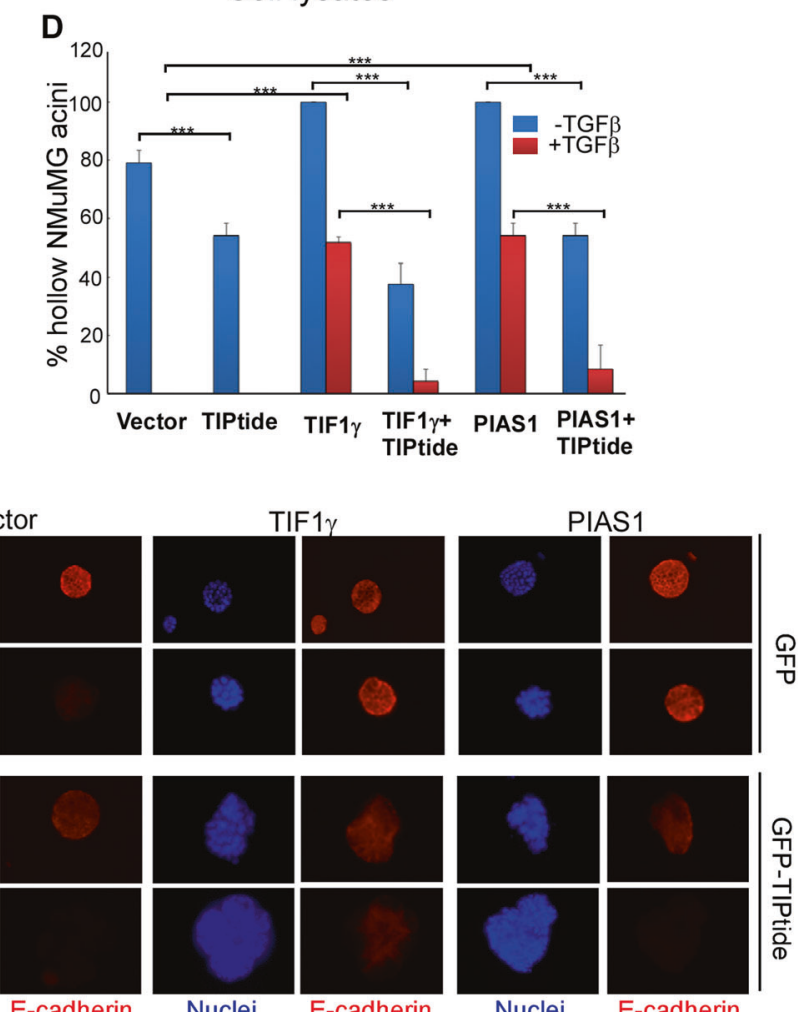

microscopy micrographs of untreated or $100 \mathrm{pM}$ TGF $\beta$-treated 8-dayold organoids derived from NMuMG cells transfected and assessed as in b. Green and red arrows indicate acinar and filled organoids, respectively. d Bar graph depicts mean \pm SEM proportion of acinar organoids expressed as a percentage of total colonies counted for each experimental condition from three biological replicates including the replicates with data shown in c. e Representative fluorescence microscopy scans of E-cadherin-(red) and nuclei-(blue) stained fixed 8-day-old three-dimensional organoids derived from NMuMG cells transfected and assessed in b, c. Statistical difference, ANOVA: $* * * P \leq 0.001$. Scale bar indicates $50 \mu \mathrm{m}$. 
A

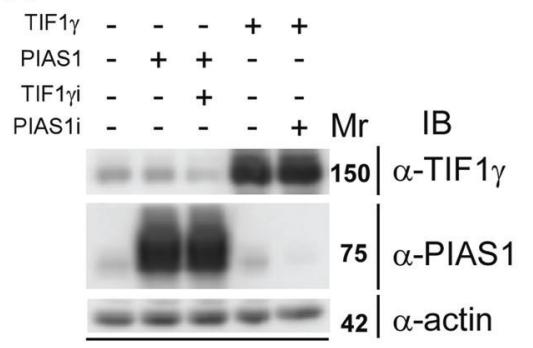

Cell lysates

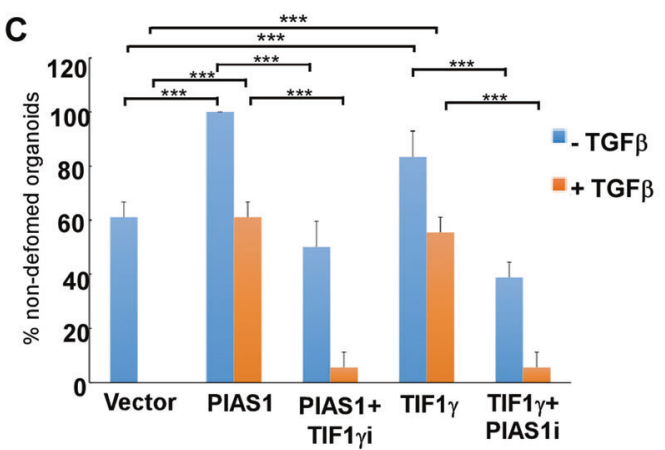

B

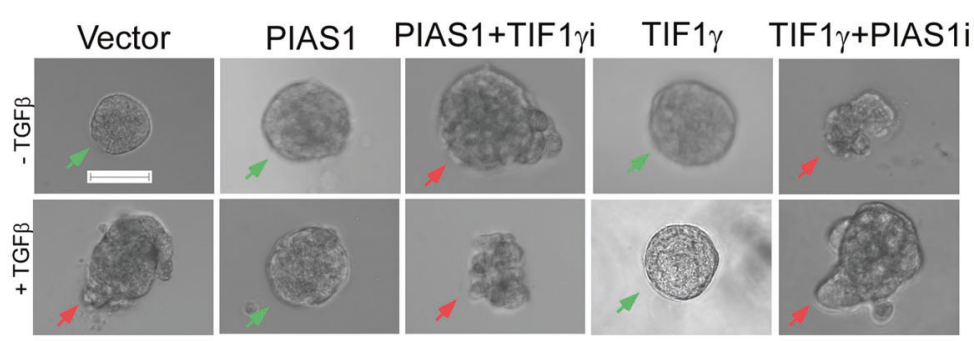

$D_{\text {FLAGTIFI } 1 \% \ldots+\ldots}+\ldots+$

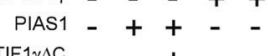

FLAGITIF1 $\gamma \Delta \mathrm{C}-\mathbf{+}+-$

PIASICS - - - + Mr IB

150 $\alpha-F L A G$
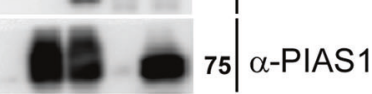

- $-0-2$ - 2 -actin

Cell lysates
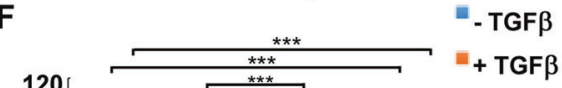

E Vector PIAS1 PIAS1+TIF1 $\gamma \Delta \mathrm{C}$ TIF1 $\gamma$ TIF1 $\gamma+\mathrm{PIAS} 1 \mathrm{CS}$
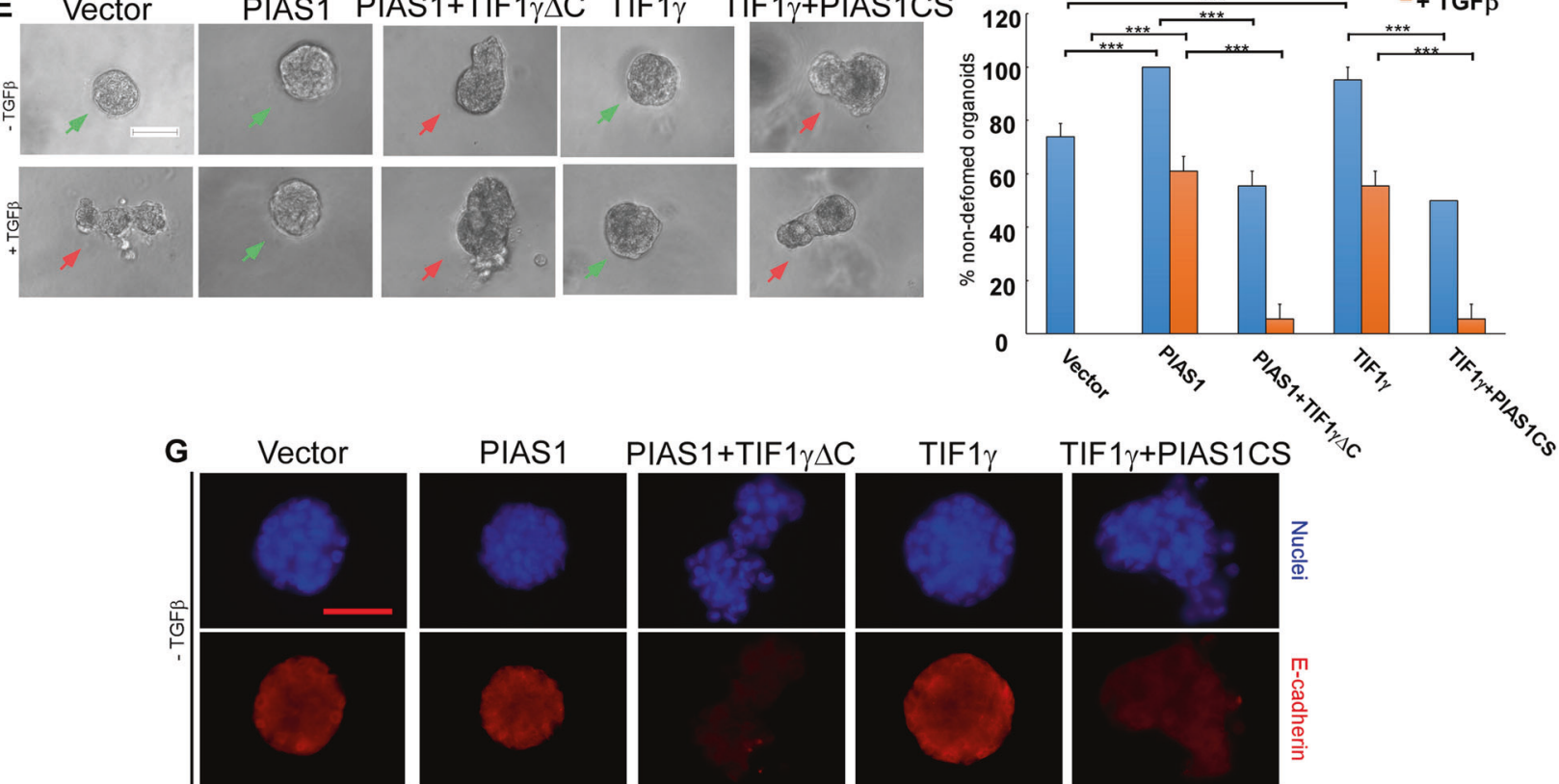

PIAS1

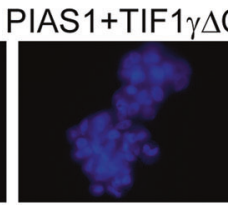

TIF1 $\gamma$
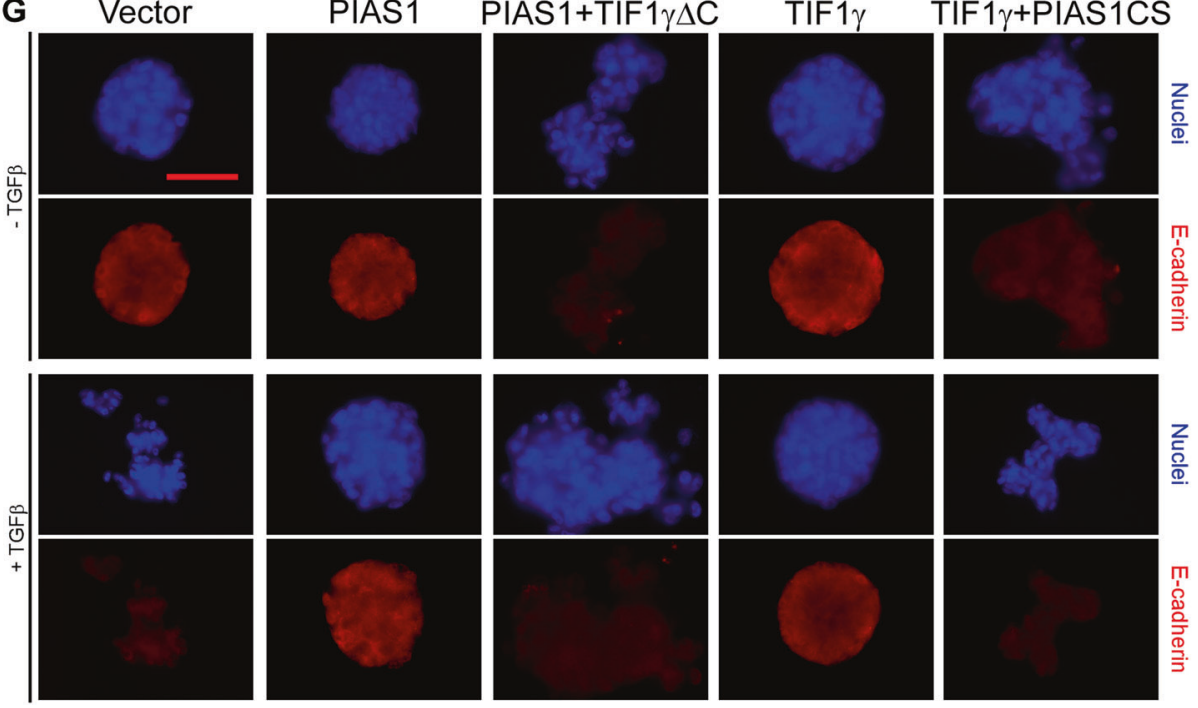

derived organoids even in the absence of TGF $\beta$, as demonstrated by lumen filling and disorganization of the acini, and E-cadherin loss and mislocalization in these organoids (Fig. 4b-e). In addition, TIPtide suppressed TIF1 $\gamma$ reversal of TGF $\beta$-induced EMT (Fig. 4b-e). Remarkably, TIPtide opposed PIAS1 suppression of 
Fig. 5 PIAS1 and TIF1 $\gamma$ reciprocally regulate TNBC cell-derived organoid invasiveness. a PIAS1, TIF $1 \gamma$, and actin immunoblotting of lysates of MDA-MB-231 cells expressing PIAS1, TIF1 $\gamma$, PIAS1i, and TIF1 $1 \gamma$ i alone or together, and which were used to generate threedimensional organoids shown in b-d. b Representative DIC light microscopy micrographs of untreated or 100pM TGF $\beta$-treated 8-dayold organoids derived from MDA-MB-231 cells transfected and assessed as in a. Green and red arrows indicate non-deformed and disrupted organoids, respectively. c Bar graph depicts mean \pm SEM proportion of non-deformed organoids expressed as a percentage of total colonies counted for each experimental condition from three biological replicates including the replicates with data shown in $\mathbf{b}$. d PIAS1, FLAG, and actin immunoblotting of lysates of MDA-MB231 cells expressing PIAS1, FLAG-TIF1 $\gamma$, PIAS1CS, and FLAG$\mathrm{TIF} 1 \gamma \Delta \mathrm{C}$ alone or together, and which were used to generate threedimensional organoids shown in e-g. e Representative DIC light microscopy micrographs of untreated or $100 \mathrm{pM}$ TGF $\beta$-treated 8-dayold organoids derived from MDA-MB-231 cells transfected and assessed as in $\mathbf{d}$. Green and red arrows indicate non-deformed and disrupted organoids, respectively. f Bar graph depicts mean \pm SEM proportion of non-deformed organoids expressed as a percentage of total colonies counted for each experimental condition from five biological replicates including the replicates with data shown in e. g Representative fluorescence microscopy scans of E-cadherin-(red) and nuclei-(blue) stained fixed 8-day-old three-dimensional organoids derived from MDA-MB-231 cells transfected and assessed in d, e. Statistical difference, ANOVA: $* * * P \leq 0.001$. Scale bar indicates $50 \mu \mathrm{m}$.

TGF $\beta$-induced EMT in the NMuMG cell-derived organoid (Fig. 4b-e). Collectively, these data suggest that the TIF1 $\gamma$-PIAS1 SUMO E3 ligase complex acts via SnoN SUMOylation to suppress EMT in the mammary epithelialderived organoid.

\section{A TIF1Y-PIAS1 SUMO E3 ligase complex regulates EMT in breast cancer cell organoids}

EMT plays key roles in cancer invasiveness and metastasis including in breast cancer [25] raising the question whether the PIAS1-TIF1 $\gamma$ SUMO E3 ligase complex plays a role in cancer. PIAS1 acts via SUMOylation of SnoN to suppress invasive behaviour of the human triple negative breast cancer MDA-MB-231 cell-derived organoids [17]. Using a threedimensional culture model system, we addressed, first, the question of whether TIF1 $\gamma$ regulates breast cancer cell invasive behaviour. MDA-MB-231 cells grown in the context of ECM, e.g., Matrigel, form multicellular structures that are mostly smooth-surfaced solid/lacking lumens sphericallyshaped organoids, a characteristic of transformed epithelial cells (Figs. 5, 6, S2, S3) $[17,18,30]$. TGF $\beta$ leads to invasiveness, budding, and disruption of the 3D-MDA-MB-231 cell-derived organoids (Figs. 5, 6, S2, S3) [17, 18, 30]. We found that knockdown of endogenous TIF $1 \gamma$ promoted disruption and invasiveness of the MDA-MB231 cell-derived organoids even in the absence of TGF $\beta$, suggesting that endogenous TIF $1 \gamma$ suppresses the invasive growth of MDAMB-231 cell-derived organoids (Fig. S2A-C). Consistently, expression of exogenous TIF1 $\gamma$ suppressed, while the SnoNinteraction defective TIF $1 \gamma \mathrm{CS}$ or the SUMO E3 ligase inactive TIF $1 \gamma \Delta \mathrm{C}$, promoted the invasive growth of MDAMB-231 cell-derived organoids (Fig. S2D-F). Knockdown of endogenous SnoN inhibited exogenous TIF1 $\gamma$ suppression of the invasive behaviour of 3D-organoids in the absence or presence of TGF $\beta$ (Fig. S2G-I). Together, these data suggested that endogenous TIF $1 \gamma$ acts in a SUMO E3 ligase and endogenous SnoN-dependent manner to suppress TGF $\beta$ induced disruptive morphogenetic behaviour of the MDAMB-231 cell-derived organoids. Accordingly, we found that expression of SUMO-SnoN suppressed the ability of knockdown of endogenous TIF $1 \gamma$ or expression of TIF $1 \gamma \Delta \mathrm{C}$ to promote disruption of organoids (Fig. S3A-F), while expression of SnoNKdR reduced TIF1 $\gamma$ suppression of the invasive phenotype of the spheroids (Fig. S3G-I). Collectively, these data show that the SUMO E3 ligase TIF1 $\gamma$ acts via SnoN SUMOylation to suppress TGF $\beta$-induced invasive growth of the triple negative breast cancer cell-derived organoids.

Next, we addressed the question of whether the TIF1 $\gamma$-PIAS1 SUMO E3 ligase complex regulates the invasive behaviour of MDA-MB-231 cell-derived organoids. Expression of exogenous PIAS1 or TIF1 $\gamma$ suppressed, while knockdown of endogenous PIAS1 or TIF1 $\gamma$ promoted the invasive growth of three-dimensional MDAMB-231 cell-derived organoids (Figs. S2, 5, and [17, 18]). Importantly, we found that knockdown of endogenous TIF $1 \gamma$ and PIAS1 abrogated exogenous PIAS1 and TIF $1 \gamma$, respectively, suppression of TGF $\beta$-induced invasive phenotype of the MDA-MB-231 cell-derived organoids (Fig. 5a-c), suggesting that endogenous PIAS1 and TIF1 $\gamma$ cooperate to suppress TGF $\beta$-induced invasive/disruptive behaviour of the MDA-MB-231 cell-derived spheroids. In other analyses, we found that expression of the SUMO E3 ligase inactive TIF $1 \gamma \Delta \mathrm{C}$ and PIAS1CS inhibited the ability of PIAS1 and TIF $1 \gamma$, respectively, to suppress TGF $\beta$ induced invasive characteristics of MDA-MB-231 cellderived organoids (Fig. 5d-f). MDA-MB-231 carcinomas represent cells displaying at least partial EMT [31], whereby the protein abundance of E-cadherin in these cells can very low to undetectable compared to other mammary epithelial and carcinoma cells [32]. Interestingly, MDA-MB-231 multicellular structures show a relatively higher protein abundance of E-cadherin as compared to 2D-cultured cells [32]. We found that expression of exogenous PIAS1 or TIF1 $\gamma$ suppressed E-cadherin loss by TGF $\beta$, which was reversed by expression of the SUMO E3 ligase inactive TIF1 $\gamma$ and PIAS1, respectively (Fig. 5g) [18]. Together, these data suggest that the TIF1 $\gamma$-PIAS1 SUMO E3 ligase complex suppresses TGF $\beta$-induced EMT, including invasive growth and E-cadherin loss, in the MDA-MB-231 cellderived organoids. 

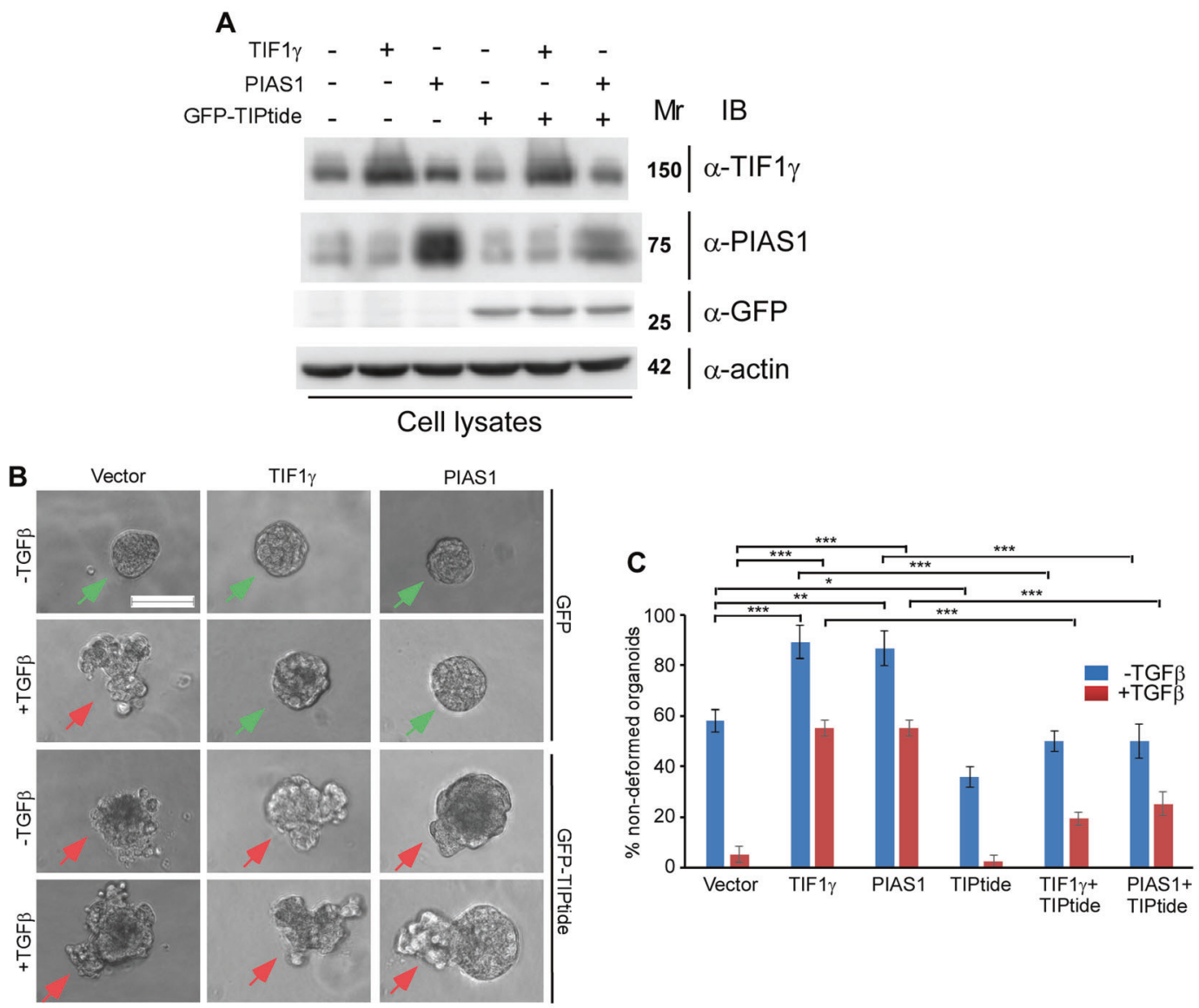

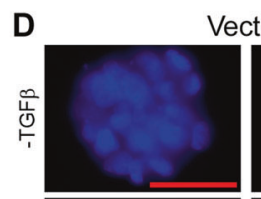

Vector
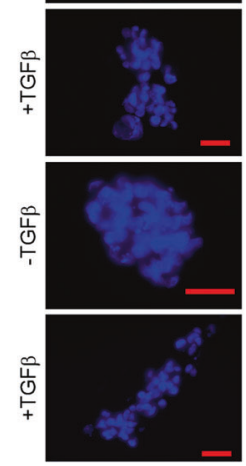

Nuclei
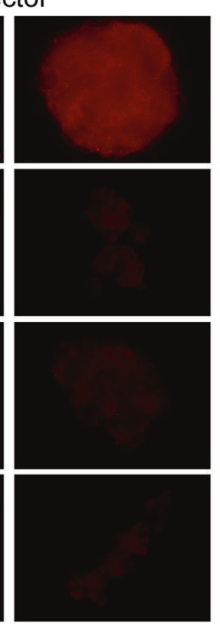

E-cadherin
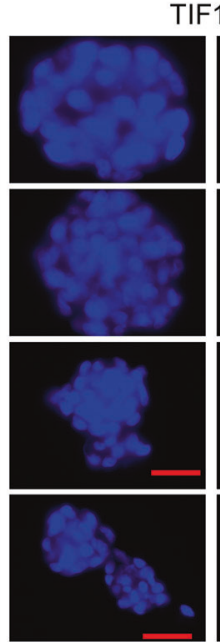

Nuclei

TIF1
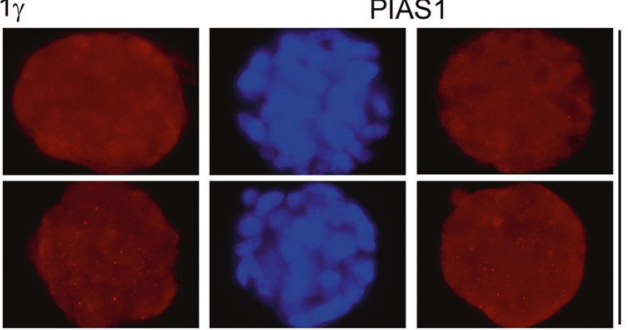

䒢

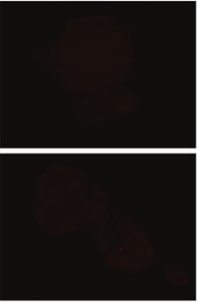

E-cadherin

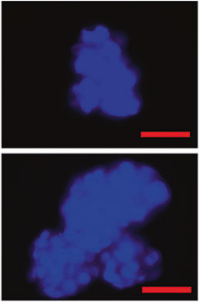

Nuclei

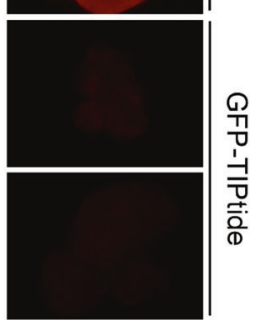

E-cadherin
Fig. 6 PIAS1 and TIF1 $\gamma$ act together via SnoN to suppress EMT in TNBC cell-derived organoids. a TIF $1 \gamma$, PIAS1, GFP, and actin immunoblotting of lysates of MDA-MB-231 cells expressing TIF1 $\gamma$, PIAS1, and GFP-TIPtide alone or together, and which were used to generate three-dimensional organoids shown in $\mathbf{b}-\mathbf{d}$. b Representative DIC light microscopy micrographs of untreated or $100 \mathrm{pM}$ TGF $\beta$-treated 8-day-old organoids derived from MDA-MB-231 cells transfected and assessed as in $\mathbf{a}$. Green and red arrows indicate nondeformed and disrupted organoids, respectively. c Bar graph depicts mean \pm SEM proportion of non-deformed organoids expressed as a percentage of total colonies counted for each experimental condition from five biological replicates including the replicates with data shown in b. d Representative fluorescence microscopy scans of E-cadherin(red) and nuclei-(blue) stained fixed 8-day-old three-dimensional organoids derived from NMuMG cells transfected and assessed in $\mathbf{b}, \mathbf{c}$. Statistical difference, ANOVA: $* P \leq 0.05 ; * * * P \leq 0.001$. Scale bar indicates $50 \mu \mathrm{m}$. The scale bar indicated in the top left image (first image) in $\mathbf{d}$ reflects the relative $50 \mu \mathrm{m}$ ruler of that image and that of other images that do not contain a scale bar. Any other images that display a different length of the $50 \mu \mathrm{m}$ scale bar reflects images that are scaled down or up relative to the first image. 

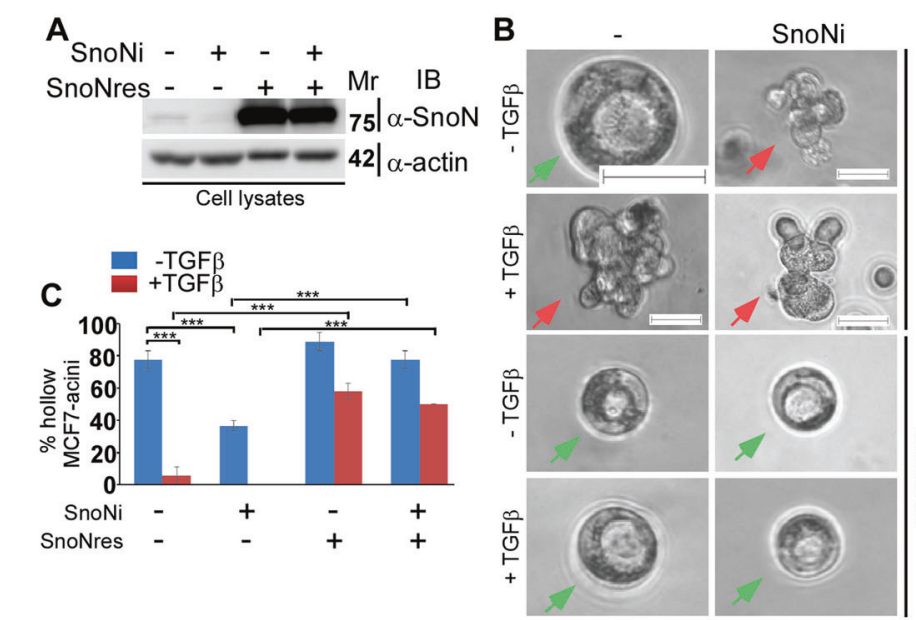

D $\quad$ - SnoNi
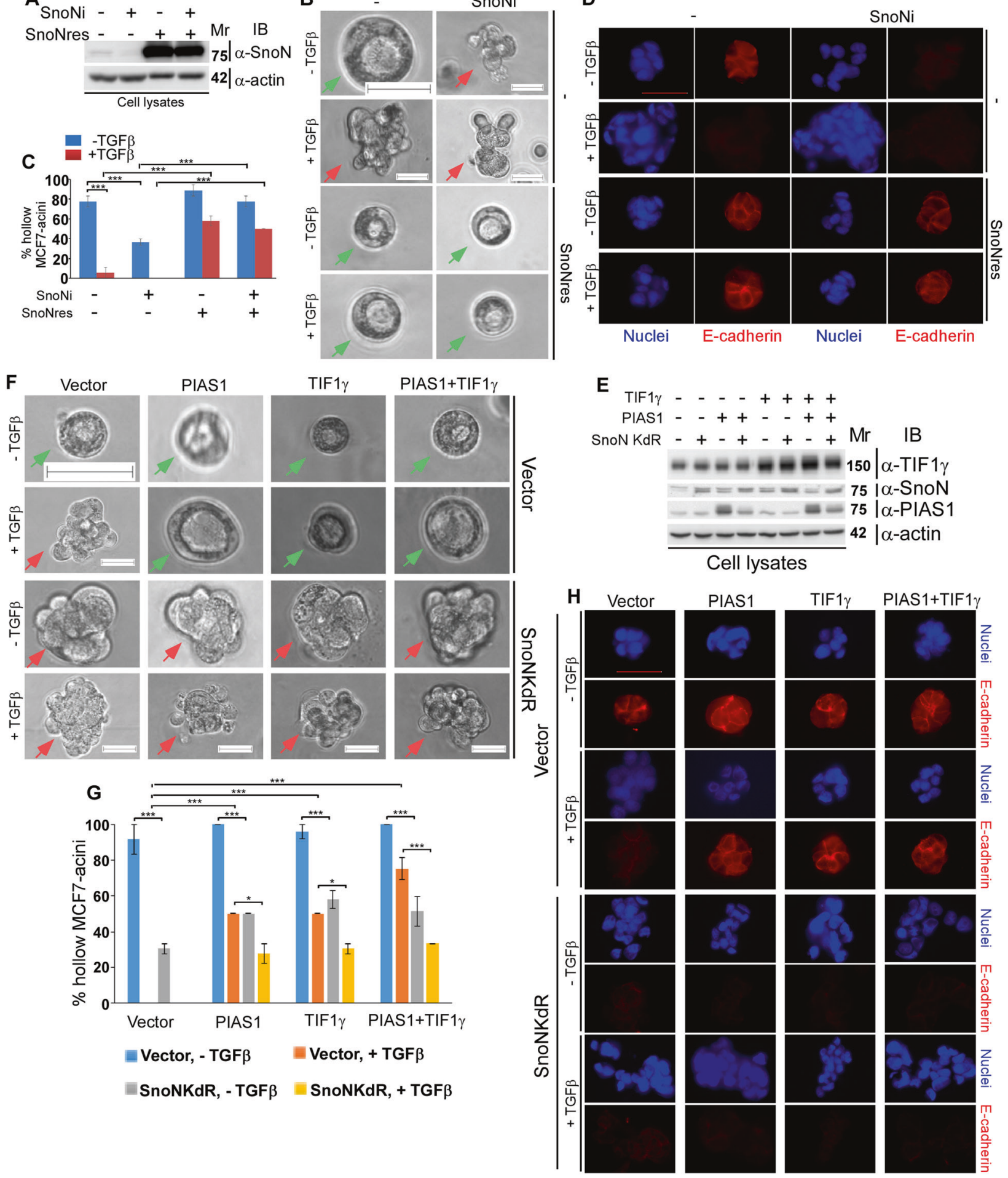

Next, we assessed the role of SnoN in mediating TIF1 $\gamma$-PIAS1-suppression of EMT in the MDA-MB-231 cell-derived organoids. We found that expression of TIPtide promoted EMT as displayed by disruptive/ deformed behaviour and loss of E-cadherin in the MDAMB-231 cell-derived organoids even in the absence of TGF $\beta$ as compared to the control (Fig. 6a-d).
Importantly, TIPtide suppressed the ability of TIF1 $\gamma$ and PIAS1 to reverse TGF $\beta$-induced EMT in the MDA-MB231 cell-derived organoids (Fig. 6a-d). Collectively, these data suggest that that the TIF $1 \gamma$-PIAS1 complex acts via SnoN SUMOylation to suppress TGF $\beta$-induced EMT-associated phenotypes in the TNBC carcinoma cell-derived organoids. 
4 Fig. 7 PIAS1-TIF1 $\gamma$ SUMO E3 ligase complex acts via SnoN SUMOylation to suppress EMT in luminal breast cancer cellderived organoids. a SnoN and actin immunoblots of lysates of MCF7 cells expressing a control vector (-) or a SnoN specific shRNA (SnoNi), with or without a SnoNi-resistant SnoN expression construct (SnoNres). b Representative DIC light microscopy micrographs of untreated or $100 \mathrm{pM}$ TGF $\beta$-treated 8-day-old organoids derived from MCF7 cells transfected and assessed as in a. Green and red arrows indicate hollow center and filled acinis, respectively. c Bar graph depicts mean \pm SEM proportion of hollow center acini expressed as a percentage of total colonies counted for each experimental condition from three biological replicates including the one with data shown in b. d Representative fluorescence microscopy scans of E-cadherin-(red) and nuclei-(blue) stained fixed 8-day-old three-dimensional organoids derived from MCF7 cells transfected and assessed in b, c. e PIAS1, TIF1 $\gamma$, SnoN, and actin immunoblots of lysates of MCF7 cells expressing PIAS1, TIF1 $\gamma$, SnoNKdR, alone or together. f Representative DIC light microscopy micrographs of untreated or $100 \mathrm{pM}$ TGF $\beta$-treated 8-day-old organoids derived from MCF7 cells transfected and assessed as in e. Green and red arrows indicate acinar and filled organoids, respectively. $\mathrm{g}$ Bar graph depicts mean \pm SEM proportion of acinar organoids expressed as a percentage of total colonies counted for each experimental condition from three biological replicates including the one with data shown in $\mathbf{f}$. $\mathbf{h}$ Representative fluorescence microscopy scans of E-cadherin-(red) and nuclei-(blue) stained fixed 8-day-old three-dimensional organoids derived from MCF7 cells transfected and assessed in $\mathbf{f}$, g. Statistical difference, ANOVA: $* P \leq 0.05 ; * * * P \leq 0.001$. Scale bar indicates $50 \mu \mathrm{m}$.

The protein abundance of PIAS1 correlated positively with survival in women with breast cancer according to a recent breast cancer tissue microarray study that included samples from luminal breast cancer subtypes [17], raising the key question whether the PIAS1-TIF1 $\gamma$ SUMO E3 complex regulates EMT in luminal breast cancer-derived cells. We used a three-dimensional model of the luminal breast cancer MCF7 cells, that express estrogen and progesterone receptors and display an epithelial phenotype, to address this question [33]. We found that Matrigel-grown MCF7 cells formed spherical-acinar type of organoids characterized by junctional localization of E-cadherin (Fig. 7b-d) [34]. TGF $\beta$ induced EMT in the MCF7 cellderived organoids characterized by filling and disruption of the organoids accompanied by reduction of the protein abundance and mislocalization of E-cadherin (Fig. 7b-d). Knockdown of endogenous SnoN induced EMT in MCF7derived organoids even in the absence of TGF $\beta$ (Fig. 7a-d), which was reversed by expression of the SnoNi-resistant SnoNres indicating that endogenous SnoN promotes an epithelial phenotype in the luminal breast cancer MCF7 cell-derived organoids. We also found that the SUMO loss of function SnoNKdR promoted, while the SUMO gain of function SUMO-SnoN suppressed EMT induction suggesting that SUMOylation is important for SnoN to inhibit TGF $\beta$-induced EMT in the MCF7 cell-derived organoids (Figs. 7e-h, S4). Consistently, the SUMO E3 ligases PIAS1 and TIF1 $\gamma$ suppressed TGF $\beta$-induced EMT, which were reversed by SnoNKdR expression (Fig. 7e-h). Conversely, reduction of the protein abundance or SUMO E3 ligase activity of PIAS1 or TIF1 $1 \gamma$ promoted EMT in MCF7 breast cancer cell-derived organoids even in the absence of TGF $\beta$, which was rescued by SUMO-SnoN expression (Fig S4A-D). Similar to NMuMG and MDA-MB231 cellderived organoids, we found that the SUMO E3 ligase inactive TIF1 $\gamma \Delta \mathrm{C}$ and PIAS1CS inhibited the ability of PIAS1 and TIF $1 \gamma$, respectively, to suppress TGF $\beta$ induced EMT of MCF7-derived organoids (Fig. 8a-d). Together, these data reveal that the PIAS1-TIF1 $\gamma$ SUMO E3 ligase complex acts via SUMOylated SnoN to suppress EMT in luminal breast cancer cell-derived organoids.

Collectively, our study reveals that the transcriptional regulator SnoN promotes the assembly of a PIAS1-TIF1 $\gamma$ SUMO E3 ligase complex that promotes its SUMOylation and ability to suppress TGF $\beta$-induced induction in organoids derived from breast epithelial and carcinoma cells. These data add insights into mechanisms by which the SUMO pathway specifies SUMO substrates for SUMOylation with important consequences for biological responses in normal and pathological conditions.

\section{Discussion}

In this study, we have discovered a novel link between the major transcriptional regulator SnoN and the SUMO pathway with implications for SnoN SUMOylation and ability to regulate the fundamental process of EMT and epithelial and carcinoma-derived tissue phenotypes. Our data reveal that SnoN promotes a protein complex containing the SUMO E3 ligases PIAS1 and TIF1 $\gamma$ that enhances SnoN SUMOylation. Functionally, our novel findings point to a co-dependency between the SUMO E3 ligases TIF1 $\gamma$ and PIAS1 in suppression of EMT induction by TGF $\beta$ and associated morphological changes of breast epithelial cells and carcinomas-tissue organoids. The idea that a SUMO E3 ligase complex can promote SUMOylation of a SUMO substrate with functional significance should advance our understanding of mechanisms that regulate protein targeting by the SUMO pathway and biological implications.

The findings revealed in this study that as a SUMO substrate, SnoN helps in the assembly of a multiprotein complex containing two SUMO E3 ligases to promote its modification by the SUMO pathway begins to address an outstanding question in the SUMO field regarding how substrate specificity and hence modification is determined given the limited number of SUMO E3 ligases as opposed to SUMO substrates identified in the literature [5]. Indeed, several SUMO substrates including p53 $[9,10,35,36]$ and Smad4 [37-39] have been described to be targeted by distinct SUMO E3 ligases raising the question of the nature of interplay between different SUMO E3 ligases targeting a selective substrate. 
A

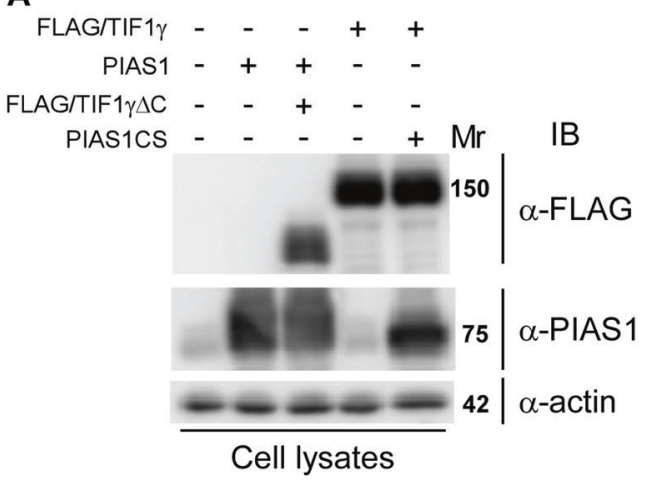

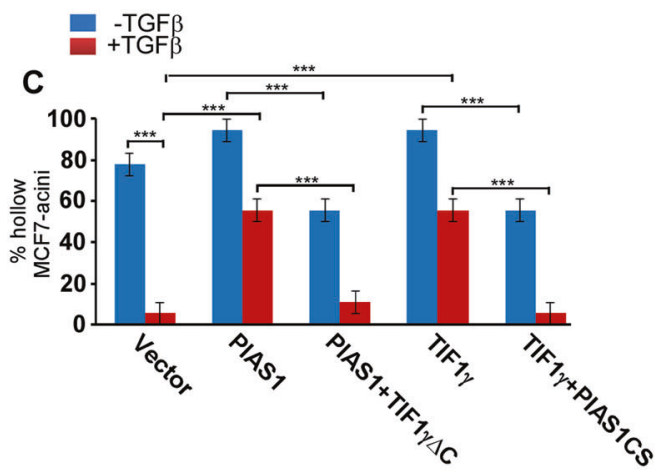

PIAS1+

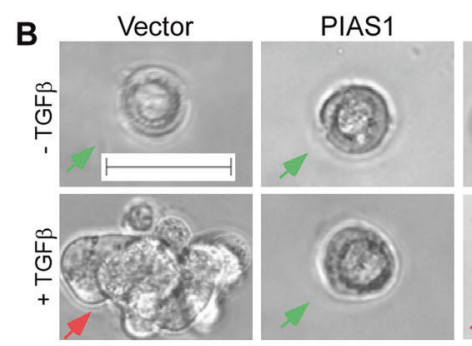

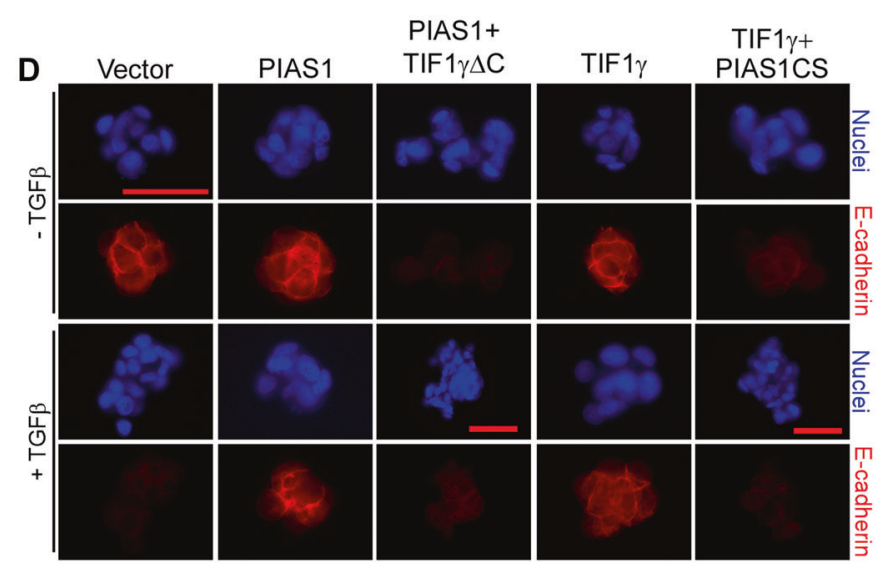

Fig. 8 PIAS1 and TIF1 $\gamma$ act reciprocally to suppress EMT in luminal breast cancer cell-derived organoids. a PIAS1, FLAG, and actin immunoblotting of lysates of MCF7 cells expressing PIAS1, FLAG-TIF1 $\gamma$, PIAS1CS, and FLAG-TIF $1 \gamma \Delta \mathrm{C}$ alone or together. b Representative DIC light microscopy micrographs of untreated or 100pM TGF $\beta$-treated 8-day-old organoids derived from MCF7 cells transfected and assessed as in a. Green and red arrows indicate acinar and filled organoids, respectively. $c$ Bar graph depicts mean \pm SEM proportion of acinar organoids expressed as a percentage of total colonies counted for each experimental condition from three biological replicates including the one with data shown in b. d Representative fluorescence microscopy scans of E-cadherin-(red) and nuclei-(blue) stained fixed 8-day-old three-dimensional organoids derived from MCF7 cells transfected and assessed in a, b. Statistical difference, ANOVA: $* * * P \leq 0.001$. Scale bar indicates $50 \mu \mathrm{m}$. The scale bar indicated in the top left image (first image) in $\mathbf{d}$ reflects the relative $50 \mu \mathrm{m}$ ruler of that image and that of other images that do not contain a scale bar. Any other images that display a different length of the $50 \mu \mathrm{m}$ scale bar reflects images that are scaled down or up relative to the first image.
SUMOylation is a ubiquitous protein modification found in multicellular organism with over 6000 protein substrates identified in human cells [40]. Different extracellular and intracellular stimuli have been suggested to alter the abundance of SUMOylated species of specific substrates with importance for normal cellular functions in development and homeostasis [5, 41]. Members of the PIAS family of SUMO E3 ligases have been reported to target multiple substrates raising the question of the implications to biological responses
$[6,7]$. Our findings that PIAS1 and TIF1 $\gamma$ coordinately enhance SnoN SUMOylation and hence suppression of EMT and associated phenotypic responses of cell-derived organoids, illuminates a regulatory mechanism by which PIAS1 controls EMT via the SUMO substrate SnoN. Our data also lead to the question of whether other members of the PIAS SUMO E3 ligase family act collaboratively with other distinct SUMO E3 ligases to promote the SUMOylation of a given substrate to regulate specific biological responses. 
The protein TIF1 $\gamma$ has been suggested to be a SUMO substrate of PIAS1, whereby SUMOylation may regulate TIF1 $\gamma$ suppression of TGF $\beta$-induced EMT in mammary cells [42]. Thus, our novel findings described in this study raise the intriguing question of whether SnoN by promoting a TIF1 $\gamma$-PIAS1 association may play a role in PIAS1's ability to promote TIF1 $\gamma$ SUMOylation. In turn, SUMOylation may promote TIF1 $\gamma$ to act as a SUMO E3 ligase for SnoN and hence EMT regulation. Addressing this potential multifaceted function of the TIF $1 \gamma-$ SnoN-PIAS 1 complex would be interesting to pursue in future studies.

Our findings that SnoN promotes a TIF1 $\gamma$-PIAS1 SUMO E3 ligase complex regulating its SUMOylation and hence EMT in mammary epithelial cells and breast carcinomas-derived tissue organoids have implications for regulation of tissue development and tumorigenesis. In future studies, it will be important to determine the effect of alteration in the abundance or activity of PIAS 1 or TIF1 $\gamma$ on developmental processes including mammary gland maturation, and tumorigenesis and progression including invasion and metastasis. Our findings also raise the key question whether the TIF1 $\gamma$-PIAS1-SnoN SUMOylation axis plays a role in epithelial cells and carcinomas derived from other types of organs including gastrointestinal tract and pancreas.

Changes in the abundance of elements of the SUMO modification machinery have been associated with pathological conditions including cancer. In addition, variations in the abundance of SUMO modified substrates have been linked to increased cancer metastasis, resistance to therapy and relapse [1, 27, 41, 43]. Thus, it will be important to determine in future studies whether perturbations in the TIF1 $\gamma$-PIAS1-SUMOylated SnoN axis identified in this study dictate cancer responses of cancer cells to chemotherapeutic and targeted therapies.

In conclusion, the findings presented in the current study uncovers novel mechanisms by which the SUMO pathway specifies and promotes the SUMOylation of protein substrates. These findings add important insights into mechanisms that control fundamental cellular processes in normal and disease conditions.

Acknowledgements We would like to thank Dr. Derrick Rancourt and Dr. Tannin Schmidt for use of the Olympus IX70 and the Olympus Fluoview FV1000 fluorescence microscopes, respectively. This work was supported by grants from the Natural Sciences and Engineering Research Council of Canada (NSERC), the Canadian Cancer Society (CCS), the Breast Cancer Society of Canada (BCSC) and the Calgary Centre for Cancer Research (CCCR) to S. Bonni, and the National Institutes of Health (NIH) - NS041021 to A. Bonni. A. Chanda is a recipient of a Charbonneau Cancer Institute Director's Award for Excellence in Research Productivity, an Eyes High International Doctoral Scholarship, a William H Davies Medical Research Scholarship, an Achievers in Medical Science (AIMS) Graduate Recruitment Scholarship, and an Eyes High Recruitment Award from the
University of Calgary. K. Karve and A. Sarkar are recipients of a Doctoral Scholarship and an Eyes High Doctoral Recruitment Scholarship, respectively, from the University of Calgary.

\section{Compliance with ethical standards}

Conflict of interest ASC is currently employed at Fog Pharma, USA. However, this study is neither funded nor associated in any manner with Fog Pharma. The other authors declare no conflict of interest.

Publisher's note Springer Nature remains neutral with regard to jurisdictional claims in published maps and institutional affiliations.

\section{References}

1. Bettermann K, Benesch M, Weis S, Haybaeck J. SUMOylation in carcinogenesis. Cancer Lett. 2012;316:113-25.

2. Palvimo JJ. PIAS proteins as regulators of small ubiquitin-related modifier (SUMO) modifications and transcription. Biochemical Soc Trans. 2007;35:1405-8.

3. Johnson ES. Protein modification by SUMO. Annu Rev Biochem. 2004;73:355-82.

4. Netherton SJ, Bonni S. Suppression of TGFbeta-induced epithelial-mesenchymal transition like phenotype by a PIAS 1 regulated sumoylation pathway in NMuMG epithelial cells. PLoS ONE. 2010;5:e13971.

5. Pichler A, Fatouros C, Lee H, Eisenhardt N. SUMO conjugation -a mechanistic view. Biomol Concepts. 2017;8:13-36.

6. Rytinki MM, Kaikkonen S, Pehkonen P, Jaaskelainen T, Palvimo JJ. PIAS proteins: pleiotropic interactors associated with SUMO. Cell Mol Life Sci. 2009;66:3029-41.

7. Rabellino A, Andreani C, Scaglioni PP. The role of PIAS SUMO E3-ligases in cancer. Cancer Res. 2017;77:1542-7.

8. Ikeuchi Y, Dadakhujaev S, Chandhoke AS, Huynh MA, Oldenborg $\mathrm{A}$, Ikeuchi $\mathrm{M}$, et al. TIF1gamma protein regulates epithelial-mesenchymal transition by operating as a small ubiquitin-like modifier (SUMO) E3 ligase for the transcriptional regulator SnoN1. J Biol Chem. 2014;289:25067-78.

9. Kahyo T, Nishida T, Yasuda H. Involvement of PIAS1 in the sumoylation of tumor suppressor p53. Mol Cell. 2001;8:713-8.

10. Wu SY, Chiang CM. p53 sumoylation: mechanistic insights from reconstitution studies. Epigenetics. 2009;4:445-51.

11. Bonni S, Bonni A. SnoN signaling in proliferating cells and postmitotic neurons. FEBS Lett. 2012;586:1977-83.

12. Deheuninck J, Luo K. Ski and SnoN, potent negative regulators of TGF-beta signaling. Cell Res. 2009;19:47-57.

13. Hsu YH, Sarker KP, Pot I, Chan A, Netherton SJ, Bonni S. Sumoylated SnoN represses transcription in a promoter-specific manner. J Biol Chem. 2006;281:33008-18.

14. Ikeuchi Y, Stegmuller J, Netherton S, Huynh MA, Masu M, Frank D, et al. A SnoN-Ccd1 pathway promotes axonal morphogenesis in the mammalian brain. J Neurosci. 2009;29:4312-21.

15. Sarker KP, Wilson SM, Bonni S. SnoN is a cell type-specific mediator of transforming growth factor-beta responses. J Biol Chem. 2005;280:13037-46.

16. Yin X, Xu C, Zheng X, Yuan H, Liu M, Qiu Y, et al. SnoN suppresses TGF-beta-induced epithelial-mesenchymal transition and invasion of bladder cancer in a TIF1gamma-dependent manner. Oncol Rep. 2016;36:1535-41.

17. Chanda A, Chan A, Deng L, Kornaga EN, Enwere EK, Morris DG, et al. Identification of the SUMO E3 ligase PIAS1 as a potential survival biomarker in breast cancer. PLoS ONE. 2017;12:e177639. 
18. Dadakhujaev S, Salazar-Arcila C, Netherton SJ, Chandhoke AS, Singla AK, Jirik FR, et al. A novel role for the SUMO E3 ligase PIAS1 in cancer metastasis. Oncoscience. 2014;1:229-40.

19. Pot I, Patel S, Deng L, Chandhoke AS, Zhang C, Bonni A, et al. Identification of a novel link between the protein kinase NDR1 and TGFbeta signaling in epithelial cells. PLoS ONE. 2013;8:e67178.

20. Kingston RE, Chen CA, Rose JK. Calcium phosphate transfection. Curr Protoc Mol Biol. 2003; Chapter 9:Unit 91.

21. Chandhoke AS, Karve K, Dadakhujaev S, Netherton S, Deng L, Bonni S. The ubiquitin ligase Smurf2 suppresses TGFbeta-induced epithelial-mesenchymal transition in a sumoylation-regulated manner. Cell Death Differ. 2016;23:876-88.

22. Bonni S, Wang HR, Causing CG, Kavsak P, Stroschein SL, Luo $\mathrm{K}$, et al. TGF-beta induces assembly of a Smad2-Smurf2 ubiquitin ligase complex that targets SnoN for degradation. Nat Cell Biol. 2001;3:587-95.

23. Blainey P, Krzywinski M, Altman N. Points of significance: replication. Nat Methods. 2014;11:879-80.

24. Barrios-Rodiles M, Brown KR, Ozdamar B, Bose R, Liu Z, Donovan RS, et al. High-throughput mapping of a dynamic signaling network in mammalian cells. Science. 2005;307:1621-5.

25. De Craene B, Berx G. Regulatory networks defining EMT during cancer initiation and progression. Nat Rev Cancer. 2013;13:97-110.

26. Ye X, Weinberg RA. Epithelial-mesenchymal plasticity: a central regulator of cancer progression. Trends Cell Biol. 2015;25:675-86.

27. Chanda A, Sarkar A, Bonni S. The SUMO System and TGFbeta signaling interplay in regulation of epithelial-mesenchymal transition: implications for cancer progression. Cancers. 2018;10:264.

28. Xu J, Lamouille S, Derynck R. TGF-beta-induced epithelial to mesenchymal transition. Cell Res. 2009;19:156-72.

29. Debnath J, Brugge JS. Modelling glandular epithelial cancers in three-dimensional cultures. Nat Rev Cancer. 2005;5:675-88.

30. Chandhoke AS, Chanda A, Karve K, Deng L, Bonni S. The PIAS3-Smurf2 sumoylation pathway suppresses breast cancer organoid invasiveness. Oncotarget. 2017;8:21001-14.

31. Wei Z, Shan Z, Shaikh ZA. Epithelial-mesenchymal transition in breast epithelial cells treated with cadmium and the role of Snail. Toxicol Appl Pharmacol. 2018;344:46-55.
32. Aiello NM, Maddipati R, Norgard RJ, Balli D, Li J, Yuan S, et al. EMT subtype influences epithelial plasticity and mode of cell migration. Dev Cell. 2018;45:681-95.e4.

33. Comsa S, Cimpean AM, Raica M. The story of MCF-7 breast cancer cell line: 40 years of experience in research. Anticancer Res. 2015;35:3147-54.

34. Krause S, Maffini MV, Soto AM, Sonnenschein C. The microenvironment determines the breast cancer cells' phenotype: organization of MCF7 cells in 3D cultures. BMC Cancer. 2010;10:263.

35. Kung CP, Khaku S, Jennis M, Zhou Y, Murphy ME. Identification of TRIML2, a novel p53 target, that enhances p53 SUMOylation and regulates the transactivation of proapoptotic genes. Mol Cancer Res. 2015;13:250-62.

36. Weger S, Hammer E, Heilbronn R. Topors acts as a SUMO-1 E3 ligase for $\mathrm{p} 53$ in vitro and in vivo. FEBS Lett. 2005;579:5007-12.

37. Lee PS, Chang C, Liu D, Derynck R. Sumoylation of Smad4, the common Smad mediator of transforming growth factor-beta family signaling. J Biol Chem. 2003;278:27853-63.

38. Long J, Wang G, He D, Liu F. Repression of Smad4 transcriptional activity by SUMO modification. Biochem. J. 2004;379: 23-9.

39. Ohshima T, Shimotohno K. Transforming growth factor-betamediated signaling via the p38 MAP kinase pathway activates Smad-dependent transcription through SUMO-1 modification of Smad4. J Biol Chem. 2003;278:50833-42.

40. Hendriks IA, Lyon D, Young C, Jensen LJ, Vertegaal AC, Nielsen ML. Site-specific mapping of the human SUMO proteome reveals co-modification with phosphorylation. Nat Struct Mol Biol. 2017;24:325-36.

41. Seeler JS, Dejean A. SUMO and the robustness of cancer. Nat Rev Cancer. 2017;17:184-97.

42. Fattet L, Ay AS, Bonneau B, Jallades L, Mikaelian I, Treilleux I, et al. TIF1gamma requires sumoylation to exert its repressive activity on TGFbeta signaling. J Cell Sci. 2013;126:3713-23.

43. Anderson DB, Zanella CA, Henley JM, Cimarosti H. Sumoylation: implications for neurodegenerative diseases. Adv Exp Med Biol. 2017;963:261-81. 